An Invitation to Toric Topology: Vertex Four of a Remarkable Tetrahedron.

Buchstaber, Victor M and Ray, Nigel

2008

MIMS EPrint: 2008.31

Manchester Institute for Mathematical Sciences

School of Mathematics

The University of Manchester

\footnotetext{
Reports available from: http://eprints.maths.manchester.ac.uk/

And by contacting: The MIMS Secretary

School of Mathematics

The University of Manchester

Manchester, M13 9PL, UK
} 


\title{
An Invitation to Toric Topology: Vertex Four of a Remarkable Tetrahedron
}

\author{
Victor M Buchstaber and Nigel Ray
}

\section{An Invitation}

Motivation. Sometime around the turn of the recent millennium, those of us in Manchester and Moscow who had been collaborating since the mid-1990s began using the term toric topology to describe our widening interests in certain well-behaved actions of the torus. Little did we realise that, within seven years, a significant international conference would be planned with the subject as its theme, and delightful Japanese hospitality at its heart.

When first asked to prepare this article, we fantasised about an authoritative and comprehensive survey; one that would lead readers carefully through the foothills above which the subject rises, and provide techniques for gaining sufficient height to glimpse its extensive mathematical vistas. All this, and more, would be illuminated by references to the wonderful Osaka lectures!

Soon afterwards, however, reality took hold, and we began to appreciate that such a task could not be completed to our satisfaction within the timescale available. Simultaneously, we understood that at least as valuable a service could be rendered to conference participants by an invitation to a wider mathematical audience - an invitation to savour the atmosphere and texture of the subject, to consider its geology and history in terms of selected examples and representative literature, to glimpse its exciting future through ongoing projects; and perhaps to locate favourite Osaka lectures within a novel conceptual framework. Thus was born the Toric Tetrahedron TT, which identifies aspects of algebraic, combinatorial, and symplectic geometry as the precursors of toric topology, and symbolises the powerful mathematical bonds between all four areas.

The Tetrahedron is the convex hull of these vertex disciplines, and every point has barycentric coordinates that measure the extent of their respective contributions. We introduce the vertices in chronological order (a mere two years separates

2000 Mathematics Subject Classification. Primary 57R19, 57S25; Secondary 14M25, 52B20, 53D20, 55P15.

Key words and phrases. Davis-Januszkiewicz space, Hamiltonian geometry, homotopy colimit, moment-angle complex, quasitoric manifold, Stanley-Reisner algebra, subspace arrangement, toric geometry, toric variety. 
second and third), not least because time has acted like a Morse function in defining a flow down the 1-skeleton, as TT has emerged from the unknown. Such flows on convex polytopes play an important rôle in toric topology! Similar geometrical analogies have suggested other useful insights as we continue to refine our understanding of the Tetrahedron.

So our primary aim is to issue a concise invitation to the study of $T T$, in which we avoid many technical details and offer an abbreviated bibliography. By way of compensation, we invite readers to sample influential publications for each of the vertex disciplines, and several of the edges and facets. We propose one or two which lie at their source, and a few important survey articles; the latter combine expert overviews with comprehensive bibliographies. Throughout our discussion we view the torus as a unifying force that maintains the integrity of the Tetrahedron, and provides a bridge between its far-flung regions. The most common context for its actions lies in the theory of manifolds, which arise repeatedly in singular, smooth, and more highly structured forms.

Before accepting our invitation, readers might also like to consult web-based resources which chart the rise of toric topology. These include: archives of Transpennine Topology Triangle meetings 18, 43, 56 and 59; the 2004 Keldysh Centenery Conference in Moscow; the Osaka meeting itself; and the Osaka City University Summer School. There is also the Manchester Toric Topology Page, and the conference page for New Horizons in Toric Topology that is scheduled to take place in Manchester during 2008. These may be found at

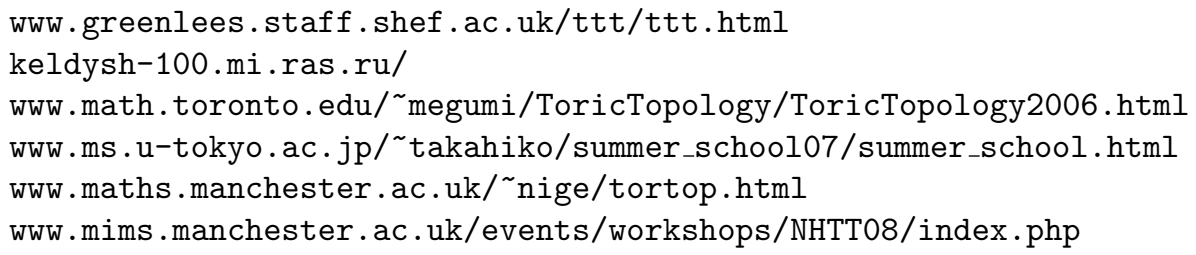

We assume that readers have a basic knowledge of algebraic topology. Nevertheless, we emphasise our convention that homology and cohomology groups $H_{*}(X)$ and $H^{*}(X)$ of a topological space $X$ are always reduced, and that their unreduced counterparts $H_{*}\left(X_{+}\right)$and $H^{*}\left(X_{+}\right)$require the addition of a disjoint basepoint. The same convention also holds for generalised theories such as cobordism and $K$-theory.

During the last 18 months, many colleagues have helped us to prepare this work (sometimes unwittingly) and we thank them all. Those who deserve special mention include Tony Bahri, Galina Buchstaber, Kostya Feldman, and Taras Panov. We apologise in advance to any whose work we have omitted or misrepresented; no doubt such sins will be brought to our attention with due speed! We are also grateful to the editors of these Proceedings and their counterparts at the AMS for all their support and encouragement.

\section{Background}

In order to present our invitation on a suitably decorative background, we offer some elementary observations on group actions. In spite of their simplicity they have been prominent in toric topology throughout its development, and have recently fed back into the original vertex disciplines to good effect. We therefore refer to them in terms that anticipate their reappearance below. 
The idea of a group $G$ acting on a set of elements $X$ has existed since Galois ushered in the revolutionary era of abstract algebra in the 1830s. The case in which $X$ denotes a set of points and $G$ a group of symmetries was studied forty years later by Klein, and proved to be an equally dramatic catalyst for the development of geometry. We draw inspiration from both points of view - in particular, we consider the poset $\mathcal{S}(G)$ of subgroups of $G$, ordered by inclusion $\leq$.

We write any $G$-set as a pair $(X, a)$, where $a: G \times X \rightarrow X$ is the function $a(g, x)=g \cdot x$ that describes the left action of symmetries $g$ on points $x$. For each $x \in X$, we denote its isotropy subgroup by $G_{x} \leq G$ and its orbit by $G x \subseteq X$; so $G / G_{x}$ and $G x$ correspond bijectively, and $G_{w}$ and $G_{x}$ are conjugate in $G$ for any $w \in G x$. The partition of $X$ into disjoint orbits is the kernel of the quotient function $q: X \rightarrow X / G$. A section for $a$ is a right inverse $s: X / G \rightarrow X$ of $q$, and is specified by choosing a preferred representative $s(G x)$ for each orbit; $s$ determines a characteristic function $\lambda_{s}: X / G \rightarrow \mathcal{S}(G)$, by $\lambda_{s}(G x)=G_{s(G x)}$.

Alternatively, suppose given a characteristic function $\lambda: Q \rightarrow \mathcal{S}(G)$ on an arbitrary set $Q$. The derived set of $\lambda$ is defined by

$$
D(\lambda)=(G \times Q) / \sim,
$$

where the equivalence relation is generated by $(g, q) \sim(h, q)$ whenever $g^{-1} h \in \lambda(q)$. So $D(\lambda)$ is a $G$-set with respect to the canonical action $g \cdot[h, q]=[g h, q]$, whose isotropy subgroups are given by $G_{[h, q]}=h \lambda(q) h^{-1}$ for any $h \in G$ and $q \in Q$. The orbits are the subsets $\{[h, q]: h \in G\}$, and projection $D(\lambda) \rightarrow Q$ onto the second factor is the associated quotient map; a canonical section is given by $s(q)=[1, q]$ for any $q \in Q$. By definition, $D(\lambda)$ is initial amongst $G$-sets $\left(X, a_{X}\right)$ equipped with functions $X \rightarrow Q$ that are constant on orbits, and sections $s_{X}: Q \rightarrow X$ such that $G_{s_{X}(q)}=\lambda(q)$ for any $q \in Q$.

Simple calculation confirms that the constructions $D(\lambda)$ and $\lambda_{s}$ are mutually inverse, and therefore that they establish a fundamental correspondence between characteristic functions and $G$-sets with sections. In particular, every choice of section $s$ for $(X, a)$ leads to a $G$-equivariant bijection

$$
f_{s}: D\left(\lambda_{s}\right) \longrightarrow X
$$

satisfying $f_{s}[g, G x]=g \cdot s(G x)$. We refer to $D\left(\lambda_{s}\right)$ as a derived form of $(X, a)$.

For any $(X, a)$ with section $s$, we partition $X / G$ by the kernel of $\lambda_{s}$, and pull the partition back to $X$ along the projection $q$. Similarly, for any characteristic function $\lambda: Q \rightarrow \mathcal{S}(G)$ we partition $Q$ by the kernel of $\lambda$, and pull the partition back to $D(\lambda)$ along projection onto the second factor. These partitions are interchanged in the obvious fashion by the fundamental correspondence, and we refer to the blocks of all four as isotropy blocks.

Now suppose that $K \unlhd G$ is a normal subgroup, and consider the surjection $\mathcal{S}(G) \rightarrow \mathcal{S}(G / K)$ of posets induced by taking quotients. Any characteristic function $\lambda: Q \rightarrow \mathcal{S}(G)$ projects to $\lambda_{K}: Q \rightarrow \mathcal{S}(G / K)$, and gives rise to a surjection $r_{K}: D(\lambda) \rightarrow D\left(\lambda_{K}\right)$; by (2.1), $r_{K}$ is equivariant with respect to the actions of $G$ and $G / K$, and the canonical section $s_{K}: Q \rightarrow D\left(\lambda_{K}\right)$ is given by $r_{K} \cdot s$. We may then interchange the rôles of $G$ and $G / K$, by starting with an epimorphism $e: H \rightarrow G$ that has kernel $L$, and lifting $\lambda$ to $\lambda^{e}: Q \rightarrow \mathcal{S}(H)$. We obtain compositions

$$
D(\lambda) \stackrel{r_{K}}{\longrightarrow} D\left(\lambda_{K}\right) \longrightarrow Q \quad \text { and } \quad D\left(\lambda^{e}\right) \stackrel{r^{e}}{\longrightarrow} D(\lambda) \longrightarrow Q
$$


which factorise and extend the original quotient function $q$ in turn. The corresponding compositions for $G$-sets are $X \rightarrow X / K \rightarrow X / G$ and $Y \rightarrow Y / L \rightarrow(Y / L) / G$.

In order to impose geometric flesh on these set theoretic bones, we proceed by assuming that $a$ is a continuous action of the topological group $G$ on a topological space $X$. Every isotropy subgroup is necessarily closed, so the characteristic map $\lambda_{s}: X / G \rightarrow \mathcal{C}(G)$ takes values in the poset of closed subgroups, and is continuous with respect to the lower topology on $\mathcal{C}(G)$, whose subbasic closed sets are of the form $H^{\uparrow}=\{J \mid H \leq J\}$. Thus $\lambda_{s}^{-1}\left(H^{\uparrow}\right)$ is closed in $X / G$, and its inverse image under $q$ is a component of the fixed point set $\operatorname{Fix}(H)$ for any closed $H \leq G$.

Alternatively, suppose given a characteristic map $\lambda: Q \rightarrow \mathcal{C}(G)$ for some topological space $Q$. The derived space $D(\lambda)$ is obtained by topologising the derived set (2.1) so that projection onto $Q$ is a quotient map; the inherited $G$-action and canonical section are then continuous, and $D(\lambda)$ is initial in the topological context.

The constructions $D(\lambda)$ and $\lambda_{s}$ are mutually inverse, and define a fundamental topological correspondence between characteristic maps and $G$-spaces with sections. In particular, every choice of continuous section for an arbitrary $G$-space $(X, a)$ leads to a $G$-equivariant homeomorphism

$$
f_{s}: D\left(\lambda_{s}\right) \longrightarrow X
$$

as before, we refer to $D\left(\lambda_{s}\right)$ as a derived form of $(X, a)$. The closures of the isotropy blocks form coverings of $X$ and $D(\lambda)$ by fixed point sets, and are identified by the fundamental correspondence. The closures of the quotient blocks in $X / G$ and $Q$ correspond similarly.

As we explore $T T$, we impose increasingly stringent geometrical conditions on $a$, whose justification and significance will become apparent. The common philosophy is to view the quotient map $q: X \rightarrow X / G$ as a singular $G$-bundle, and to interpret the geometry of $X$ in terms of $G$ and $X / G$ using an appropriate derived form. This approach has a long and distinguished history in equivariant topology, and features in attempts such as those of Jänich [62] and Davis [34] to classify Lie group actions on smooth manifolds.

One additional aspect of the topological situation is important, namely the interplay between homotopy theory and the action $a$. For any topological group $G$ there exist various functorial models for a contractible space $E G$ on which $G$ acts freely, and with closed orbits; any such $E G$ is final amongst well-behaved free $G$-spaces and $G$-equivariant homotopy classes of maps. The quotient map $E G \rightarrow E G / G$ is then a universal principal $G$-bundle, where $E G / G=B G$ is a classifying space for $G$, and unique up to homotopy equivalence.

Any $G$-space $X$ may be replaced by the homotopy equivalent $E G \times X$, on which $G$ acts freely by $g \cdot(e, x)=(g \cdot e, g \cdot x)$. So projection onto the first factor represents the homotopy class of maps to the final object, and classifies a principal $G$ bundle over the quotient $E G \times_{G} X$. The latter space is the Borel construction on $(X, a)$, otherwise known as the homotopy quotient, and is equivalent to $X / G$ when $G$ acts freely on $X$. In other cases, $E G \times_{G} X$ has superior topological properties to $X / G$, and toric topology deals as much with homotopy quotients as with orbit spaces.

These considerations illustrate the power of algebraic topology to generalise constructions that are purely algebraic. Whenever $G$ is discrete, for example, the cohomology ring $H^{*}(B G ; \mathbb{Z})$ is isomorphic to the group theoretic cohomology of $G$. In consequence, Milnor's original version [74] of $B G$ stimulated the development of continuous cohomology theory for topological groups. 
Henceforth, we restrict $G$ to a compact $n$-dimensional torus $T^{n}$ unless otherwise stated, on the grounds that toroidal symmetry is implicit in many physical systems because the coordinates may be thought of as angles. We sometimes assume that $X$ is an object of a category that is particularly convenient for homotopy theory, such as the $k$-spaces of $[\mathbf{9 6}]$.

Examples. We now invite readers to consider three families of examples that illustrate our background principles in action. The first is a local model for many others, and the second and third are generalised and extended later. All three are based on the coordinatewise multiplication map $\mu: \mathbb{C}^{n} \times \mathbb{C}^{n} \rightarrow \mathbb{C}^{n}$, defined by

$$
\mu\left(y_{1}, \ldots, y_{n}, z_{1}, \ldots, z_{n}\right)=\left(y_{1} z_{1}, \ldots, y_{n} z_{n}\right)
$$

for any $n>0$. We abbreviate the set $\{1, \ldots, n\}$ to $[n]$, and write the subspace $\left\{z \mid z_{i}=0\right.$ for $\left.i \notin \omega\right\}$ as $\mathbb{C}^{\omega} \leq \mathbb{C}^{n}$ for any subset $\omega \subseteq[n]$. Similarly, we denote the compact torus $\left(S^{1}\right)^{\omega}$ by $T^{\omega} \subset\left(\mathbb{C}^{\times}\right)^{\omega}$, as a subspace of $\left\{z \mid z_{i} \neq 0\right\} \subset \mathbb{C}^{\omega}$. Finally, we write $\mathbb{C}_{\delta}$ and $T_{\delta}<\mathbb{C}^{n}$ for the diagonal line and its unit subcircle respectively.

ExAmple 2.1. Let $X$ be $\mathbb{C}^{n}$, and $a: T^{n} \times \mathbb{C}^{n} \rightarrow \mathbb{C}^{n}$ the restriction of $\mu$. For any $z \in \mathbb{C}^{n}$, the isotropy subgroup $T_{z}^{n}$ is $T^{[n] \backslash \omega}$, where $\omega$ is the unique subset of $[n]$ for which $z \in\left(\mathbb{C}^{\times}\right)^{\omega}$; the orbit $T^{n} z$ is the $|\omega|$-dimensional torus $T^{\omega} z$. There is a homeomorphism $h: \mathbb{C}^{n} / T^{n} \rightarrow \mathbb{R}_{\geqslant}^{n}$ to the non-negative coordinate cone, induced by the formula

$$
h\left(z_{1}, \ldots, z_{n}\right)=\left(\left|z_{1}\right|^{2}, \ldots,\left|z_{n}\right|^{2}\right),
$$

and the standard inclusion of $\mathbb{R}^{n}$ in $\mathbb{C}^{n}$ specifies a canonical section $s: \mathbb{R}_{\geqslant}^{n} \rightarrow \mathbb{C}^{n}$. The isotropy blocks are the subspaces $\left(\mathbb{C}^{\times}\right)^{\omega} \subset \mathbb{C}^{n}$, whose closures $\mathbb{C}^{\omega}$ are the fixed point sets $\operatorname{Fix}\left(T^{[n] \backslash \omega}\right)$. The blocks project to the interiors of the corresponding faces $\mathbb{R}_{\geqslant}^{\omega}$ of the polyhedron $\mathbb{R}_{\geqslant}^{n}$, whose closures are the faces themselves. The characteristic map $\lambda_{s}: \mathbb{R}_{\geqslant}^{n} \rightarrow \mathcal{S}\left(T^{n}\right)$ assigns the subtorus $T^{[n] \backslash \omega}$ to the interior of the face $\mathbb{R}_{\geqslant}^{\omega}$, and the derived form associated to $s$ is the canonical homeomorphism

$$
f_{s}:\left(T^{n} \times \mathbb{R}_{\geqslant}^{n}\right) / \sim \longrightarrow \mathbb{C}^{n} .
$$

EXAMPLE 2.2. For any ordered pair $(p, q)$ of positive integers, let $Z(p, q)$ be the product of unit spheres $S^{2 p+1} \times S^{2 q+1} \subset \mathbb{C}^{p+q+2}$ and $a$ the product action of $T^{p+1} \times T^{q+1}$. The orbit space is homeomorphic to the product $\Delta^{p} \times \Delta^{q}$ of standard simplices in $\mathbb{R}_{\geqslant}^{p+1} \times \mathbb{R}_{\geqslant}^{q+1}$, by (2.3). Let $K$ be the 2 -torus $T(p, q)<T^{p+1} \times T^{q+1}$ of points

$$
\left(\left(t_{1}, \ldots, t_{1}\right),\left(t_{2}, \ldots, t_{2}, t_{1}^{-1} t_{2}\right)\right),
$$

where $t_{1}, t_{2} \in S^{1}$. Then $M(p, q)=Z(p, q) / T(p, q)$ is a 2 nd stage Dobrinskaya tower [40], and is a $2(p+q)$-dimensional smooth manifold, equipped with an action of the quotient $(p+q)$-torus $T^{p+q+2} / T(p, q)$. The associated factorisation $(2.2)$ takes the form $Z(p, q) \rightarrow M(p, q) \rightarrow \Delta^{p} \times \Delta^{q}$; many homotopic sections exist for the projection, because the product of simplices is contractible.

The isotropy blocks of $\Delta^{p} \times \Delta^{q}$ corresponding to subtori $T^{p+q-k}$ are the relative interiors of the $k$-dimensional faces, for $0 \leq k \leq p+q$; their closures are the faces themselves. The corresponding components of the fixed point sets in $M(p, q)$ are lower-dimensional 1 st or 2 nd stage towers. The coordinates induced on $M(p, q)$ take the form $\left(x_{1}, \ldots, x_{n} ; u_{1}, \ldots, u_{p}\right)$, where $x$ lies in $\Delta^{p} \times \Delta^{q}$ and $u$ in $T^{p+q}$; they are singular over the boundary. All these structures on $M(p, q)$ lift to naturally to their counterparts in $S^{2 p+1} \times S^{2 q+1}$. 
By construction, $M(p, q)$ is the projectivisation of the complex $(q+1)$-plane bundle $\mathbb{C}^{q} \oplus \eta$ over $\mathbb{C} P^{p}$, where $\eta$ is the canonical line bundle. So $M(1,1)$ is a $2 n d$ stage Bott tower [53], and is diffeomorphic to a Hirzebruch surface; it is also the bounded flag manifold $B_{2}[\mathbf{2 4}]$, consisting of flags $0<L_{1}<L_{2}<\mathbb{C}^{3}$ for which $L_{2}$ contains the first coordinate line; then $(u, v) \in T^{2}$ acts on $L_{1}$ by $(1, v, u v)$, and on $L_{2}$ by $(1,1, u)$. The case $q=0$ reduces to the quotient space $S^{2 p+1} / T_{\delta}$, and is diffeomorphic to $\mathbb{C} P^{p}$.

In fact $M(p, q)$ may be extended to a $k$ th stage tower, by iterating the projectivisation procedure, and replacing $\mathbb{C}^{q} \oplus \eta$ with a sequence of more general bundles. The details are provided by Dobrinskaya [40], and require care. The corresponding bounded flag manifolds $B_{k}$ are significant contributors to complex cobordism theory [23], [88], as we shall explain below.

ExAmple 2.3. Let $X$ be $\mathbb{C}^{n+1} \backslash 0$, and $a: \mathbb{C}_{\delta}^{\times} \times\left(\mathbb{C}^{n+1} \backslash 0\right) \rightarrow\left(\mathbb{C}^{n+1} \backslash 0\right)$ the restriction of $\mu$. The quotient space is the algebraic variety $\mathbb{C} P^{n}$, on which the algebraic torus $\left(\mathbb{C}^{\times}\right)^{n}$ acts with a single dense principal orbit. In this context, the toric coordinates identify $\mathbb{C} P^{n}$ with an equivariant compactification of $\left(\mathbb{C}^{\times}\right)^{n}$.

\section{The Toric Triangle}

We now invite readers to focus on the oldest facet of the Toric Tetrahedron, which we label $A C S$ and call the Toric Triangle. Each of the vertices $A, C$, and $S$ represents appropriate aspects of a familiar discipline, whose key ideas and selected literature we introduce in this section. The edges of the triangle are populated with interdisciplinary work that involves two of the vertices in some proportion, and interior points represent activity that combines all three.

The first vertex $\boldsymbol{A}$. This is the original vertex of the Triangle, and represents algebraic geometry; or more specifically, the study of toric varieties. These appear to have been introduced in 1970 by Demazure [37], whose work is therefore the source of the entire Toric Tetrahedron. Demazure's constructions also became known as torus embeddings, and an early survey was provided by Danilov [33] in 1978. Twenty years later, Cox $[\mathbf{3 0}]$ popularised the term toric geometry for the expanding array of ideas that surrounded the vertex, and updated his survey in 2002 to take account of developments in more distant regions of $T T[\mathbf{3 1}]$.

The seminal books of Ewald [44], Fulton [50], and Oda [82] presented the topic to readers with little background in algebraic geometry, and showed singular examples to be as important as those that are smooth; indeed, the term toric manifold is sometimes used to distinguish the latter. For the sake of brevity, we restrict attention to compact toric varieties, whether singular or smooth.

An essential ingredient of the toric geometer's worldview is the concept of a complete fan (which we abbreviate to fan henceforth). Every fan arises from $m>n$ vectors in the integral lattice $\mathbb{Z}^{n}<\mathbb{R}^{n}$, which determine $m$ positive half-lines known as rays; the rays intersect the unit sphere $S^{n-1} \subset \mathbb{R}^{n}$ in $m$ points. Initially, we insist that these be distributed so as to form the vertices of a simplicial subdivision $K_{\Sigma}$, none of whose faces contains antipodal points. The set of convex polyhedra obtained by taking the infinite cone on each face of $K_{\Sigma}$, with vertex the origin, is then a simplicial fan $\Sigma$. By convention, $\{0\}$ is included as the cone on the empty face $\varnothing$. If we allow the cones to be spanned by linearly dependent rays, we obtain 
a less restricted notion of fan that is of sufficient generality for our purposes below; in every case, a fan decomposes $\mathbb{R}^{n}$ as a union of closed cones.

For any fan $\Sigma$ in $\mathbb{R}^{n}$, we may construct a compact toric variety $X_{\Sigma}$. It is covered by affine varieties $X_{\sigma}$ as $\sigma$ ranges over the cones of $\Sigma$, where $X_{\sigma}$ and $X_{\sigma^{\prime}}$ are glued along $X_{\tau}$ whenever $\sigma$ and $\sigma^{\prime}$ have a common subcone $\tau$. The algebraic torus $\left(\mathbb{C}^{\times}\right)^{n}$ acts compatibly on the $X_{\sigma}$, and therefore on $X_{\Sigma}$; in particular $X_{\{0\}}$ is naturally isomorphic to $\left(\mathbb{C}^{\times}\right)^{n}$, and the action is by multiplication. Every cone contains the subcone $\{0\}$, so $X_{\{0\}}$ is dense in $X_{\Sigma}$ and forms the principal orbit of the global action. There is also a natural map of posets from the cones of $\Sigma$ to the subtori of $\left(\mathbb{C}^{\times}\right)^{n}$, ordered by inclusion. The construction of $X_{\Sigma}$ in this fashion is tantamount to using the fan as a combinatorial blueprint for compactifying the principal orbit $\left(\mathbb{C}^{\times}\right)^{n}$ in such a way that coordinatewise multiplication extends to a $\left(\mathbb{C}^{\times}\right)^{n}$-action. This viewpoint has been extremely influential during the growth of toric geometry.

Associating $X_{\Sigma}$ to $\Sigma$ actually establishes a fundamental varietal correspondence between general fans and compact toric varieties, and the algebraic properties of the fan are reflected in the geometry of the corresponding variety. If $\Sigma$ is simplicial, for example, then the singularities of $X_{\Sigma}$ are homeomorphic to finite quotients of $\mathbb{R}^{2 n}$, and the variety is an orbifold. If every cone is defined by rays that extend to a basis of $\mathbb{Z}^{n}$, then $\Sigma$ is regular, and $X_{\Sigma}$ is smooth; and if $K_{\Sigma}$ is the boundary of a convex simplicial polytope, then $\Sigma$ is polytopal, and $X_{\Sigma}$ is projective.

One of Demazure's motivations for introducing toric varieties was his interest in their algebraic isomorphisms, and he proved that $\operatorname{Aut}\left(X_{\Sigma}\right)$ is a compact Lie group in any nonsingular case, with maximal torus $T^{n}<\left(\mathbb{C}^{\times}\right)^{n}$ acting by restriction. Subsequently, his results were extended to toric orbifolds by Cox [29], and to the general case by Bühler.

The orbit space $Q_{\Sigma}$ of the maximal torus may be identified with a polyhedral ball in $\mathbb{R}^{n}$, whose bounding sphere is subdivided by the dual of $\Sigma$. Every subspace $X_{\sigma}$ projects onto the dual of the corresponding face of $K_{\Sigma}$; in particular, $X_{\{0\}}$ projects onto the interior of the ball. Following Fulton [50, §4.1], for example, the underlying topological $T^{n}$-space of $X_{\Sigma}$ may then be expressed in derived form by means of a homeomorphism

$$
f_{\Sigma}:\left(T^{n} \times Q_{\Sigma}\right) / \sim \longrightarrow X_{\Sigma}
$$

The image of the associated section is known to algebraic geometers as a canonical submanifold with corners, possibly singular, of $X_{\Sigma}$ [82, §1.3]. If $\Sigma$ is polytopal, then $Q_{\Sigma}$ is the corresponding simple polytope.

EXAMPLE 3.1. The simplest example of a toric manifold is, of course, given by $\mathbb{C} P^{n}$, whose fan may be taken to be the $n+1$ vectors $e_{1}, \ldots, e_{n},-\sum_{j=1}^{n} e_{j}$ in $\mathbb{R}^{n}$; it is regular and polytopal, being normal to the $n$-simplex on $0, e_{1}, \ldots, e_{n}$. More generally, the vectors $e_{1}, \ldots, e_{n},-w \sum_{j=1}^{n} e_{j}$ form a fan $\Sigma$ for any nonnegative integer $w$; it is simplicial, but regular only when $w=1$. For $w>1$, the corresponding toric variety is an orbifold $\mathbb{C} P^{n}(w, 1, \ldots, 1)$, known as a weighted projective space, whereas the orbit space $Q_{\Sigma}$ is an $n$-simplex for all values of $w$.

We describe generalisations of $\mathbb{C} P^{n}(w, 1, \ldots, 1)$ in $\S 5$. 
The second vertex $\boldsymbol{C}$. The study of geometrical objects such as regular polyhedra forms one of the oldest branches of mathematics, and reaches back thousands of years. Nevertheless, we invite readers to consider combinatorial geometry as the second vertex of $T T$, on the grounds that we shall focus on those aspects of the subject that have come of age since connections with toric varieties were first discovered. The edge $A C$ was exposed in spectacular fashion by Stanley's solution [91] to one half of McMullen's conjecture in 1980; however brief, his work is the obvious source for the emergence and development of $C$ as an independent vertex.

Two of the most influential surveys of polytope theory have been Grübaum's book [54], and Ziegler's more recent lectures of 1995 [99]. The latter is now quoted by authors working in many areas of $T T$, and emphasised the principle that simple polytopes are by far the most amenable to general discussion. More specific to the vertex $C$ itself is Stanley's key text [92], where he transformed traditional invariants of polytope theory into powerful algebraic machinery for the study of abstract simplicial complexes $K$. His pivotal construction was the face ring $R[K]$, otherwise known as the Stanley-Reisner algebra of $K$, over a commutative ring $R$.

Whenever possible we write the vertices of $K$ as $v_{1}, \ldots, v_{m}$, and the vertex set as $V$; we also assume that the faces $\sigma \subseteq V$ include the empty face $\varnothing$. So $\sigma \in K$ and $\rho \subseteq \sigma$ imply that $\rho \in K$. For algebraic purposes we often insist that the vertices are graded by real dimension 2, and rewrite subsets $\omega \subset V$ as squarefree monomials $\prod_{\omega} v_{j}$ in the polynomial algebra $S_{\mathbb{Z}}(V)$ on the $v_{j}$. The face ring of $K$ is then defined as the quotient $S_{R}(V) /(\omega \notin K)$, and the set of monomials divisible by faces of $K$ forms an additive $R$-basis.

In order to place McMullen's conjecture in context, we recall that the dual, or polar polytope $P^{*}$, of any simple polytope $P$ is simplicial, and therefore bounded by a simplicial sphere $K_{P}$. For example, the $n$-cube $I^{n}$ is simple, and its polar is the cross-polytope, whose boundary $K_{I^{n}}$ triangulates $S^{n-1}$. This is also the duality of (3.1), because $K_{P}$ defines the normal fan of $P$, whose rays may be taken to be integral by small deformations of $P$ if necessary.

Historically, integral vectors $f(P)$ and $h(P)$ were defined for simple polytopes in terms of $K_{P}$, as follows. For any simplicial complex $K$ of dimension $n-1$, let $f_{j}$ denote the number of faces of dimension $j$, for $0 \leq j \leq n-1$, and write $f(K)$ for the vector $\left(f_{0}, \ldots, f_{n-1}\right)$; let $f_{-1}=1$ count the empty face. Then define integers $h_{j}$ for $0 \leq j \leq n$ by the polynomial equation $\sum_{j=0}^{n} h_{j} t^{n-j}=\sum_{j=-1}^{n-1} f_{j}(t-1)^{n-1-j}$, and write $h(K)$ for the vector $\left(h_{0}, \ldots, h_{n}\right)$. Finally, let the $g$-vector $g(K)$ be given by $\left(g_{0}, \ldots, g_{[n / 2]}\right)$, where $g_{0}=1$ and $g_{j}=h_{j}-h_{j-1}$. In case $K=K_{P}$, there is a beautiful Morse theoretic argument [17] involving flow along the 1-skeleton of $P$ to show that the Dehn-Sommerville equations

$$
h_{j}=h_{n-j} \quad \text { for } \quad 0 \leq j \leq n
$$

always hold. The case $h_{n}=h_{0}$ is Euler's equation $\sum_{j=1}^{n-1}(-1)^{j} f_{j}=1+(-1)^{n-1}$.

Since 1959 [90] it has been possible to characterise integral vectors that realise $f(K)$ for some simplicial complex $K$. The corresponding problem for simplicial polytopes has a longer history, and the correct formulation was only achieved when McMullen stated his conjecture in 1971 [73]. Given an integral vector $h$, he proposed two requirements: firstly, that the Dehn-Sommerville equations (3.2) hold; and secondly, that the associated $g$-vector is of a combinatorial form referred to 
by Stanley as an $M$-vector. Stanley confirmed the necessity of McMullen's conditions, by proving that $g(P)$ is an $M$-vector for any simple polytope $P$. Sufficiency was established simultaneously by Billera and Lee [13], using completely different methods. McMullen's characterisation is now known as the $g$-theorem.

The revolutionary aspect of Stanley's proof was his use of the toric variety $X_{\Sigma}$ corresponding to the fan defined by $K_{P}$. By construction, $\Sigma$ is both simplicial and polytopal, so $X_{\Sigma}$ is a projective toric orbifold. The Danilov-Jurkiewicz theorem therefore describes its rational cohomology ring by means of an isomorphism

$$
\mathbb{Q}\left[K_{P}\right] / J \longrightarrow H^{*}\left(X_{\Sigma} ; \mathbb{Q}\right),
$$

where $J$ denotes an ideal generated by linear terms associated to the rays of $\Sigma$. If $\Sigma$ is regular, the integral version of (3.3) also describes the cohomology ring $H^{*}\left(X_{\Sigma} ; \mathbb{Z}\right)$. Stanley applied the hard Lefschetz theorem to $H^{*}\left(X_{\Sigma} ; \mathbb{Q}\right)$, and deduced the existence of a 2-dimensional cohomology class $w$ with certain multiplicative properties. His proof concluded by showing that the coordinates $g_{j}$ of the $g$-vector measure the ranks of the $2 j$-dimensional components of the quotient ring $H^{*}\left(X_{\Sigma} ; \mathbb{Q}\right) /(w)$, and therefore that $g$ is an $M$-vector. In this context, the Dehn-Sommerville equations are equivalent to rational Poincaré duality for $X_{\Sigma}$.

The Dehn-Sommerville equations actually remain true for a considerably wider class of simplicial complexes, whereas restrictions on $g$-vectors are less easy to extend. For example, the conjecture that $g(K)$ is an $M$-vector for every simplicial sphere $K$ appears still to be open. Similar mystery shrouds the $f$-vectors of convex polytopes; an explicit classification was given by Steinitz [93] for dimension 3 in 1906, yet the situation for dimension 4 remains obscure [100]!

Since Stanley's initial applications, face rings with particular algebraic properties such as Cohen-Macauley or Gorenstein have become important test-beds for commutative algebra [18], and interest has grown in identifying simplicial complexes $K$ for which $\mathbb{Z}[K]$ is of a specific type. Relevant results include Reisner's Theorem [89], which supplies homological criteria for $K$ to be Cohen-Macaulay; his criteria are satisfied by all simplicial spheres.

For any simplicial complex $K$, the face poset $\mathcal{L}(K)$ contains the faces of $K$, ordered by inclusion; the empty face is a minimal element, and the combinatorial type of $K$ is determined by the isomorphism class of $(K)$. By analogy, the combinatorial type of an arbitrary polytope $P$ is defined to be the isomorphism class of its face poset $\mathcal{L}(P)$, which omits the empty face but includes the maximal face $P$ itself. So for any simple polytope $P$, the opposite poset $\mathcal{L}^{o p}(P)$ is isomorphic to the face poset $\mathcal{L}\left(K_{P}\right)$. The $f-, g$-, and $h$-vectors of $P$ are combinatorial invariants, because they depend solely upon $\mathcal{L}(P)$, as do our applications to toric topology below. We therefore deal only with combinatorial polytopes henceforth, on the understanding that they may be represented by face posets or affine realisations as the occasion demands. We emphasise that $n$-dimensional polytopes in $\mathbb{R}^{n}$ may be combinatorially equivalent, but not affinely isomorphic.

The third vertex $S$. The origins of symplectic structure may also be traced back down the years, as far as the profound reformulation of Newtonian mechanics that was begun by Lagrange, and continued by Hamilton during the first half of the 19th century. Hamilton's choice of coordinates $q$ and $p$ for phase space, representing position and momentum respectively, led eventually to the notion of a symplectic manifold $(M, \omega)$. The phase space $M$ is smooth, and $\omega$ is a closed 
non-degenerate 2-form, whose existence forces the dimension of $M$ to be even. Typical examples of symplectic manifolds include the total space of any cotangent bundle, which admits a canonical $\omega$, and an arbitrary Kähler manifold, equipped with the imaginary part of the defining metric. The former example illustrates the importance of noncompact phase spaces.

Many mechanical systems admit groups of symmetries, which may be interpreted as diffeomorphisms of $M^{2 n}$; in the symplectic setting such symmetries preserve the 2-form, and are known as symplectomorphisms. Experience with classical systems such as pendulums and spinning tops suggests that effective Hamiltonian actions of a compact torus $T^{k}$ are important special cases, which require $k \leq n$ and determine a moment map $\Phi: M \rightarrow \mathbb{R}^{k}$. Here $\mathbb{R}^{k}$ is realised as the dual Lie algebra of $T^{k}$, and $\Phi$ is constant on the $T^{k}$-orbits. When the phase space is compact, Atiyah [3] and Guillemin and Sternberg [56] proved in 1982 that the image $\Phi(M)$ is always a convex polytope. These works are the source of the third vertex $S$, whose associated discipline is the symplectic geometry of toroidal symmetry, moment maps, and convex polytopes.

The second edition [6] of Audin's book offers an inspirational survey of developments up to 2004. The overarching theme is Hamiltonian actions of tori, with special attention being paid to the half-dimensional case; in this situation, the real-valued coordinate functions of $\Phi$ form a completely integrable system, and the associated torus action is called completely integrable. It follows that the fixed point set of any such action is nonempty, and that its elements are isolated.

An important technique in Hamiltonian geometry is that of symplectic reduction, which compensates for the fact that the quotient of a symplectic manifold $(M, \omega)$ by a Hamiltonian action of $T^{k}$ need not be symplectic. Reduction involves forming the level submanifold $Z=\Phi^{-1}(x)$ of a regular value of the moment map, and imposing a symplectic structure on the quotient $Z / T^{k}$, which is better behaved, and at worst an orbifold. For each factorisation of $T^{k}$ into a product of subtori, the reduction may be performed in corresponding stages. Symplectic reduction has played an important rôle in fully understanding the relationship between the vertices $A$ and $S$ of the Tetrahedron, but enters rarely into our discussions below. We therefore refer interested readers to $[\mathbf{6}]$ for further details.

The edge $\boldsymbol{A} \boldsymbol{C}$. After the existence of a wider toric world was revealed in [91], the edge $A C$ was quickly colonised. One pioneer was Khovanskii, whose work [64], [65] resonated with that of Stanley by introducing torus actions into problems where none were initially apparent.

Every monomial in $n$ complex variables $z_{1}, \ldots, z_{n}$ may be written as $z^{\alpha}$ for some integral vector $\left(\alpha_{1}, \ldots, \alpha_{n}\right)$. So any Laurent polynomial $f(z)=\sum c_{\alpha} z^{\alpha}$ defines a finite set $A \subset \mathbb{R}^{n}$ containing those $\alpha$ for which the coefficient $c_{\alpha} \in \mathbb{C}$ is nonzero, and the Newton polyhedron of $f$ is the convex hull of $A$. Now suppose that $W_{f} \subset\left(\mathbb{C}^{\times}\right)^{n}$ is the complete intersection defined by a non-degenerate system $f_{1}(z)=\cdots=f_{k}(z)=0$ of $k \leq n$ polynomial equations, with respective Newton polyhedra $\Delta_{1}, \ldots, \Delta_{k}$. Khovanskii considered the problem of computing invariants such as the arithmetic genus $\chi\left(W_{f}\right)$ in terms of the number of integral points interior to the $\Delta_{j}$; his results generalised Kushnirenko's theorem [6] for the case $k=n$, where $W_{f}$ is discrete. He proceeded by forming the normal fan $\Sigma$ of the Minkowski sum of the $\Delta_{j}$, interpreted the corresponding toric variety $X_{\Sigma}$ as the compactification of $\left(\mathbb{C}^{\times}\right)^{n}$, and proved that the closure $\operatorname{cl}\left(W_{f}\right) \subset X_{\Sigma}$ is nonsingular 
and conveniently embedded. He completed his computation by applying Ehler's description [43] of the Chern classes of a nonsingular toric variety (now settled on the edge $A T$ ). Additional details may be found in $[\mathbf{5 0}, \S 5]$, for example.

A more unconventional topic on this edge was furnished by work of Sturmfels and Sullivant [94], who considered the cut polytope $\mathrm{Cut}^{\square}(G)$ associated to an arbitrary finite graph $G=(V, E)$, which is well-known in combinatorial optimization. It is rarely simple for larger graphs, so the projective toric variety $X_{G}$ determined by its normal fan is usually singular. The authors carried out experimental calculations in the ring $H^{*}\left(X_{G}: \mathbb{C}\right)$, and discussed applications to algebraic statistics.

The edge $\boldsymbol{A S}$. The importance of the edge $A S$ was demonstrated in 1988 by Delzant's characterisation [36] of non-singular projective toric varieties in terms of symplectic geometry. For any $n$-polytope $P$ with regular normal fan $\Sigma(P)$, he invested the toric variety $X_{\Sigma(P)}$ with a symplectic form for which the action of $T^{n}$ is completely integrable, and deduced that the image of the associated moment map recovers $P$. His proof proceeded by identifying $X_{\Sigma(P)}$ with the symplectic reduction associated to the moment map $\Phi: \mathbb{C}^{m} \rightarrow \mathbb{R}^{m-n}$ of a certain $(m-n)$-dimensional subtorus $K<T^{m}$ that depends upon $P$. Delzant also established the uniqueness of his construction, by showing that any two compact, connected symplectic manifolds with completely integrable torus actions are Hamiltonian diffeomorphic whenever their moment maps have the same image.

The edge $\boldsymbol{C S}$. The existence of the edge $C S$ emphasises the rôle of the moment map in linking classical examples of convex polytopes to the phase spaces of interesting mechanical systems. We shall discuss examples with a topological component in Section 5 .

We may also interpret Delzant's classification theorem as a fundamental symplectic correspondence between primitive polytopes and symplectic manifolds with completely integrable actions. This has been extended to weighted polytopes and symplectic toric orbifolds by Lerman and Tolman [69].

The facet $\boldsymbol{A C S}$. A major occupant of this facet is the study of arrangements $A=\left\{L_{1}, \ldots, L_{r}\right\}$ of subspaces $L_{j}<\mathbb{C}^{m}$, which began before the advent of toric geometry, and may now be interpreted as a limit of activity within the interior of TT. The source is Arnold's calculation of the integral cohomology ring of the coloured braid group [2] in 1969, which stimulated a rapidly expanding interest in arrangements and their complements $\mathbb{C}^{m} \backslash\left(\bigcup_{j} L_{j}\right)$, denoted by $U(A)$. Because they arise as configuration spaces of various classical mechanical systems, their properties are central to many problems in algebraic, combinatorial, and symplectic geometry. An extensive general survey was produced by Björner [14] in 1994, and several other overviews are available.

In 1988 Goresky and MacPherson [51] determined the additive structure of $H^{*}(U(A) ; \mathbb{Z})$, as a direct sum of homology groups associated to the intersection poset $\mathcal{I}(A)$. Subsequently, Cox [30, Theorem 4.1] showed how to interpret Delzant's constructions in terms of symplectic structure on the complements of a particular class of complex arrangements; these are now of major importance to toric topology, and will also be discussed in Section 5 . 


\section{Vertex Four - Toric Topology}

Overview. Many phenomena associated with the Toric Triangle refer to topological spaces that are equipped with additional geometric structure, and it is natural to search for their analogues in a purely topological context. Such results are the substance of toric topology, our fourth vertex $T$, whose convex hull with $A C S$ forms the Toric Tetrahedron.

The pioneering work is due to Davis and Januszkiewicz in 1991, whose paper $[35]$ is the source of toric topology. They introduced a class of well-behaved actions $a$ of $T^{n}$ on $2 n$-dimensional manifolds $M$ that are more amenable to topological analysis than those of toric geometry. Here we make the additional assumption that $M$ and $a$ are smooth, although such considerations and their consequences were left to readers in [35]. The pairs $(M, a)$ are now known as quasitoric manifolds, and are defined by two conditions. The first is a weakening of the varietal structure by requiring only that $a$ be locally standard, and so ensures that $M / T^{n}$ is a manifold with corners; the second identifies the orbit space as a simple polytope $P^{n}$. Since $P^{n}$ is contractible, $a$ admits many smooth sections.

The faces of the polytope are the closures of its isotropy blocks, amongst which the $m$ facets $F_{j}$ are distinguished by their dimension $n-1$, and by the isotropy subcircles $T\left(F_{j}\right)<T^{n}$ associated to their interiors, for $1 \leq j \leq m$. Because $P^{n}$ is simple, every face $G$ of dimension $n-d$ is a unique intersection $\cap_{k=1}^{d} F_{j_{k}}$ of $d$ facets; the isotropy subtorus $T(G)<T^{n}$ associated to its interior has rank $d$, and is the isomorphic image of the product $\times_{k=1}^{d} T\left(F_{j_{k}}\right)$. The facets lift to closed facial submanifolds $X_{j} \subset M$ of dimension $2(n-1)$, which are fixed by the circles $T\left(F_{j}\right)$ for $1 \leq j \leq m$. The $X_{j}$ intersect transversally, and $G$ lifts to the closed submanifold $\cap_{k=1}^{d} X_{j_{k}}$ of dimension $2(n-d)$, which we abbreviate to $X(G) \subset M$; it is fixed by the $d$-torus $T(G)$.

Alternatively, suppose given a simple polytope $P^{n}$ with $m$ facets $F_{j}$, and a characteristic map $\lambda: P^{n} \rightarrow \mathcal{T}\left(T^{n}\right)$ to the poset of subtori. Assume that the kernel of $\lambda$ partitions $P^{n}$ by the interiors of its faces, and associates a circle $T_{\lambda}\left(F_{j}\right)<T^{n}$ to the interior of the facet $F_{j}$, for $1 \leq j \leq m$. Finally, suppose that the product map $\times_{k=1}^{d} T_{\lambda}\left(F_{j_{k}}\right) \rightarrow T^{n}$ is actually an isomorphism onto its image $T_{\lambda}(G)$ for every face $G=\cap_{k=1}^{d} F_{j_{k}}$ of $P$; this is condition (*) of Davis and Januszkiewicz, which guarantees that $\lambda$ is determined by the circles $T_{\lambda}\left(F_{j}\right)$.

The derived manifold of $\lambda$ is given by topologising (2.1) as

$$
M(\lambda)=\left(T^{n} \times P^{n}\right) / \sim,
$$

where $(g, p) \sim(h, p)$ whenever $g^{-1} h \in \lambda(p)$, and imposing the natural smooth structure suggested by Davis [34]. The canonical $T^{n}$-action and the section $s(q)=[1, q]$ are also smooth, and $X_{\lambda}(G)=\left(T^{n} / T_{\lambda}(G) \times G\right) / \sim$ is a $2(n-d)$-dimensional submanifold for any face $G$; it is fixed by the $d$-torus $T_{\lambda}(G)$. Conversely, any quasitoric manifold $(M, a)$ with smooth section $s$ admits a characteristic map $\lambda_{s}: P^{n} \rightarrow \mathcal{T}\left(T^{n}\right)$, where $\lambda_{s}(p)=T(G)$ for any $p$ in the interior of $G$.

These constructions are mutually inverse, and determine a fundamental quasitoric correspondence between characteristic maps and quasitoric manifolds. In particular, every choice of smooth section $s$ for an arbitrary quasitoric manifold $(M, a)$ leads to a $T^{n}$-equivariant homeomorphism

$$
f_{s}: M\left(\lambda_{s}\right) \longrightarrow M,
$$


which may be upgraded to a diffeomorphism by appeal to [34]. As in [24], we refer to $M\left(\lambda_{s}\right)$ as the derived form of $(M, a)$.

The derived manifold (4.1) is a version of Vinberg's construction [95], as adapted by Davis and Januszkiewicz. The characteristic map depends only on the combinatorial type of $P$, and may be reformulated in terms of face posets as an order reversing map $\lambda: \mathcal{L}\left(P^{n}\right) \rightarrow \mathcal{T}\left(T^{n}\right)$, or an order preserving map $\lambda: \mathcal{L}\left(K_{P}\right) \rightarrow \mathcal{T}\left(T^{n}\right)$. In the latter context, condition (*) specifies the values of $\lambda$ on arbitrary faces of $K_{P}$ in terms of those on its $m$ vertices.

Whenever such $\lambda$ exist, we deem $P^{n}$ and $K_{P}$ to be supportive, and describe the associated $M(\lambda)$ as lying over $P^{n}$. But there are many unsupportive simple polytopes! An intriguing family of examples arises when $K_{P}$ is the $n$-dimensional cyclic polytope $C_{k}^{n}[\mathbf{9 9}]$, for any number $k$ of vertices satisfying $k \geq 2^{n}$; in other words, no quasitoric manifolds can lie over the dual polytopes.

In pursuing their cohomological calculations, Davis and Januszkiewicz defined an auxiliary space $D J(K)$ for arbitrary simplicial complexes $K$, and proved the seminal fact that $H^{*}\left(D J(K)_{+} ; \mathbb{Z}\right)$ realises the Stanley-Reisner algebra $\mathbb{Z}[K]$. Whenever $P$ supports a quasitoric manifold $(M, a)$, they exhibited an isomorphism

$$
\mathbb{Z}\left[K_{P}\right] / J \longrightarrow H^{*}\left(M_{+} ; \mathbb{Z}\right)
$$

[35, Theorem 4.14], where $J$ is the ideal generated by the first Chern classes of $n$ complex line bundles over $D J\left(K_{P}\right)$, associated to the isotropy subcircles of $a$. This is the toric topologist's version of the Danilov-Jurkiewicz theorem (3.3) for toric varieties. It arises from the fibration

$$
M \longrightarrow D J\left(K_{P}\right) \longrightarrow B T^{n},
$$

which is built into Davis and Januszkiewicz's model for $D J\left(K_{P}\right)$ as the homotopy quotient $E T^{n} \times_{T^{n}} M$; the homotopy type of the latter does not depend on the choice of $(M, a)$. Philosophically, $D J\left(K_{P}\right)$ is the homotopy theorists' substitute for $P^{n}$, whose $T^{n}$-bundle $E T^{n} \times M \rightarrow D J\left(K_{P}\right)$ desingularises the quotient map.

The construction of the second auxiliary space $\mathcal{Z}_{K}$ in [35] was equally significant, and closely related to that of the derived manifold (4.1). It admits a canonical action $a$ of $T^{m}$, whose orbit space $P_{K}$ is the simple polyhedral complex dual to $K$; if $K=K_{P}$ then $P_{K}=P^{n}$ for any simple polytope. When $K_{P}$ is supportive, every characteristic map $\lambda: P_{K} \rightarrow \mathcal{T}\left(T^{n}\right)$ satisfying condition $(*)$ determines a subtorus $K(\lambda)<T^{m}$ of dimension $m-n$ that acts freely on $\mathcal{Z}_{K}$. The quotient $n$-torus $T^{m} / K(\lambda)$ acts on the orbit space $\mathcal{Z}_{K} / T^{m-n}$, which is readily identifiable with the derived quasitoric manifold $M(\lambda)$. By varying the choice of $\lambda$, we run through a family of subtori $K(\lambda)<T^{m}$ in the poset $\mathcal{T}(T)$, and the corresponding factorisations $Z_{K} \rightarrow M(\lambda) \rightarrow P^{n}$ display all possible quasitoric manifolds over $P^{n}$ in the form of (2.2). From this point of view, the homotopy quotient $E T^{m} \times_{T^{m}} \mathcal{Z}_{K}$ is a natural model for $D J\left(K_{P}\right)$, and may be identified with any $E T^{n} \times_{T^{n}} M(\lambda)$ by factoring out the freely acting subtorus $K(\lambda)$.

The next major impact on the subject was made in 2002 by the survey of Buchstaber and Panov [20], which contains a wealth of fascinating extensions, generalisations, and applications of the ideas of [35]. Several of these were responsible for establishing new regions of $T T$, and are introduced in the context of the appropriate edges and faces below. So far as the vertex $T$ is concerned, Buchstaber and Panov emphasised the importance of working with arbitrary simplicial complexes $K$, and constructed both $D J(K)$ and the moment-angle complex $\mathcal{Z}_{K}$ by more direct 
and functorial methods, as unions of subspaces indexed by the faces of $K$. These were developed in [86] as diagrams over the face category $\operatorname{CAT}(K)$, whose objects are the faces of $K$ and morphisms their inclusions. In particular, $\mathcal{Z}_{K}$ and $D J(K)$ were identified as homotopy colimits of certain exponential diagrams, which now underlie a homotopy theoretic industrial zone at the vertex $T$.

Highlights. We have reached the most seductive passage of our invitation! As the Toric Tetrahedron has acquired form and substance, new areas of toric topology have been revealed, spawning examples, insights, and results that illuminate many topological phenomena. We present a small selection of our favourites.

1) Non-quasitoric manifolds. There is considerable insight to be gained from understanding why certain families of likely-looking manifolds cannot be quasitoric.

We focus on the Milnor hypersurfaces $H_{j, k}$, for $1 \leq j \leq k$. By definition, $H_{j, k} \subset \mathbb{C} P^{j} \times \mathbb{C} P^{k}$ is the complex hypersurface dual to the tensor product $\eta_{1} \otimes \eta_{2}$ of the canonical line bundles over the respective factors. It has dimension $2(j+k-1)$, and may be realised by projectivising the stabilisation $\eta_{1}^{\perp} \oplus \mathbb{C}^{k-j}$ of the orthogonal complement of $\eta_{1}$ over $\mathbb{C} P^{j}$. So its integral cohomology ring takes the form

$$
\mathbb{Z}[x, y] /\left(x^{j+1}, y^{k}+y^{k-1} x+\cdots+y^{k-j} x^{j}\right),
$$

where $x$ is the pullback of the generator of $H^{2}\left(\mathbb{C} P^{j} ; \mathbb{Z}\right)$ and $y \in H^{2}\left(H_{j, k} ; \mathbb{Z}\right)$ is the first Chern class of the canonical line bundle along the fibre. If $H_{j, k}$ is quasitoric then its cohomology ring takes the form (4.2), and $y^{k-j}\left(y^{j}+y^{j-1} x+\cdots+x^{j}\right)$ must lie in an ideal of $H^{*}\left(H_{j, k} ; \mathbb{Z}\right)$ generated by squarefree monomials and linear factors. A straightforward calculation shows that this is impossible; so $H_{j, k}$ admits no suitable action of $T^{j+k-1}$.

By way of comparison, consider the $2(j+k-1)$-dimensional quasitoric manifold $B_{j, k}$ constructed in [23]. It is the quotient of $\left(S^{3}\right)^{j} \times S^{2 k-1}$ by the $(j+1)-$ dimensional subtorus $T(j, k)<T^{2 j+k}$ of points

$$
\left\{\left(t_{1}, \ldots, t_{j}, t_{j+1}, \ldots, t_{j+1}\right): t_{i} \in T \text { for } 1 \leq i \leq j+1\right\}
$$

thus $T(j, k)$ acts freely. Projection onto $\left(S^{3}\right)^{j}$ shows that $B_{j, k}$ is the projectivisation of the complex $k$-plane bundle $\gamma_{j}^{\perp} \oplus \mathbb{C}^{k-j}$ over the bounded flag manifold $B_{j}$ of Example 2.2. So its integral cohomology ring takes the form

$$
\mathbb{Z}\left[x_{1}, \ldots, x_{j}, y\right] /\left(x_{i}\left(x_{i}-x_{i-1}\right), y^{k}+y^{k-1} x_{j}+\cdots+y^{k-j} x_{j}^{j}: 1 \leq i \leq j\right),
$$

where $x_{i}$ is the pullback of the eponymous element in $\left.H^{2}\left(B_{j}\right) ; \mathbb{Z}\right)$, and $x_{0}=0$. A short calculation reveals that $y^{k-j}\left(y^{j}+y^{j-1} x_{j}+\cdots+x_{j}^{j}\right)$ now factorises as

$$
y^{k-j}\left(y+x_{1}\right)\left(y-x_{1}+x_{2}\right) \ldots\left(y-x_{j-1}+x_{j}\right)
$$

in $H^{2 j}\left(B_{j, k} ; \mathbb{Z}\right)$. Note that $\gamma_{j}$ is the pullback of $\eta$ along its classifying map, which induces a monomorphism from (4.3) to (4.4) by mapping $x$ to $x_{j}$ and fixing $y$.

2) Stably complex structures. Davis and Januszkiewic [35, §6] showed that every quasitoric manifold admits a stably complex structure, but did not remark that many inequivalent choices are possible. On bounded flag manifolds $B_{n}$, for example, some structures bound and others do not [27], with serious consequences for complex cobordism. To be precise, we invest $(M, a)$ with an omniorientation [24], by choosing orientations for $M$ itself, and for every facial submanifold $X_{j}$; so there are $2^{m+1}$ omniorientations in total. The normal 2-plane bundle of every embedding $X_{j} \subset M$ is also oriented, and extends to a complex line bundle $\sigma_{j}$ over 
$M$ for $1 \leq j \leq m$, by the Pontryagin-Thom construction. An omniorientation gives rise to a unique isomorphism $\tau(M) \oplus \mathbb{C}^{m-n} \cong \oplus_{j=1}^{m} \sigma_{j}$, and hence to a canonical stably complex structure on $M$.

The additional structure is captured by a fundamental omnioriented correspondence, between omnioriented quasitoric manifolds and dicharacteristic homomorphisms $\ell: T^{\mathcal{F}} \rightarrow T^{n}$, where $\mathcal{F}$ denotes the set of $m>n$ facets of an oriented combinatorial polytope $P^{n}$, and $\ell$ obeys the analogue of condition $(*)$. There are $2^{m}$ such homomorphisms $\ell$ for every characteristic map $\lambda: P^{n} \rightarrow \mathcal{T}\left(T^{n}\right)$, because any isotropy circle $\lambda(p)=T(F)$ admits two isomorphisms $T_{F} \rightarrow T(F)$ from the coordinate subcircle $T^{F}$; there is one for each orientation of $T(F)$, corresponding to the orientations of the $X(F) \subset M(\lambda)$. Also, $\ell$ is represented on Lie algebras by an integral $n \times m$ matrix, which may be refined to $\left[I_{n}: \Lambda\right]$ for some $n \times(m-n)$ matrix $\Lambda$, by careful choice of bases. We label the pair $(P, \Lambda)$ as the quasitoric combinatorial data $[\mathbf{2 2}]$ underlying the omnioriented quasitoric manifold $M(\ell)$.

ExAmple 4.1. In the case of $\mathbb{C} P^{3}$, the polytope $P$ is $\Delta^{3}$ and $n$ is 3 ; so $m-n=1$ and $\Lambda$ is a 3 -dimensional column vector. For the omniorientation induced by the standard toric varietal structure, this vector is $(-1,-1,-1)^{t}$, whereas one of the 32 alternatives yields $(+1,-1,+1)^{t}$. All three Chern numbers are nonzero in the first case, but zero in the second.

For the Dobrinskaya tower $M(3,3)$ of Example 2.2, $P$ is $\Delta^{3} \times \Delta^{3}$ and $n$ is 6 ;

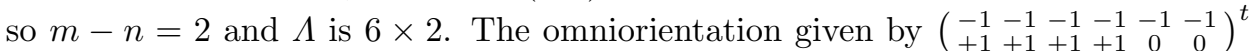
is induced by a toric varietal structure, and 3 of the $2^{9}$ alternatives are given by negating one or both of the columns.

3) Complex cobordism. The Thom spectrum $M U$ is universal amongst complex oriented ring spectra, and is one of the most important objects in stable homotopy theory. Complex bordism $\Omega_{*}^{U}(\cdot)$ and cobordism $\Omega_{U}^{*}(\cdot)$ are the associated homology and cohomology theories, and may be defined in terms of stably complex manifolds as explained by Conner and Floyd [28] and Quillen [87] respectively. The coefficient ring $\pi_{*}(M U)$ is isomorphic to $\Omega_{*}^{U}\left(S^{0}\right)$, and abbreviated to $\Omega_{*}^{U}$; this is the celebrated complex cobordism ring, shown by Milnor [75] and Novikov [78] to be isomorphic to a graded polynomial algebra $\mathbb{Z}\left[z_{n}: n \geq 1\right]$, where $z_{n}$ has dimension $2 n$. Sum and product of cobordism classes are induced by disjoint union and cartesian product of manifolds respectively. No truly canonical choice of polynomial generators is known, although $z_{p-1}$ may be represented by $\mathbb{C} P^{p-1}$ for any prime $p$; the remaining $z_{n}$ are represented by linear combinations of the Milnor hypersurfaces $H_{j, k}$, where $j+k-1=n$. Such results are obtained by calculating Chern numbers, which completely determine complex cobordism classes.

The essential feature of [23] was to exhibit omniorientations of the $B_{j, k}$ to act as substitutes for the Milnor hypersurfaces. It follows that every complex cobordism class may be represented by a disjoint union of quasitoric manifolds. The connected analogue is considerably more subtle, and relies on the construction of connected sums that are compatible with omniorientations; this was finally achieved in [22], where every class of dimension greater than 2 is represented by an omnioriented quasitoric manifold. The analogous problem for connected algebraic varieties remains unsolved, having been posed by Hirzebruch in 1958 .

The value of these ideas lies in the principle that quasitoric representatives $\left(M^{2 n}, a\right)$ offer an alternative source of discrete data for any complex cobordism class. The fundamental omnioriented correspondence, for example, gives rise to 
quasitoric combinatorial data $(P, \Lambda)$, whereas the isolated fixed points of any $T^{n}$ action give rise to classical local invariants; theoretically, we may express the latter in terms of the former. A crucial case is that of the sign $\sigma(x)= \pm 1$ of any fixed point $x$. Its expression in terms of combinatorial data was obtained by Panov [83], and compares the global orientation of $M$ with the orientation of the tangent space $T_{x}(M)$ induced by the $n$ incident facial submanifolds. Signs are therefore indispensable for formulating the connected sum of quasitoric manifolds [22]. Alternatively, $\sigma(x)$ compares the orientation underlying the stably complex structure on $M$ with that induced on $T_{x}(M)$ by the $T^{n}$-action; so $\sigma(x)$ is always positive when $M$ is complex or almost complex.

The quasitoric manifolds $B_{n}$ were introduced to complex cobordism theory in $1986[\mathbf{8 8}]$, as iterated sphere bundles with bounding omniorienations. They were shown to represent the basis elements $b_{n}$ for $\Omega_{*}^{U}\left(\mathbb{C} P^{\infty}\right)$, dual to powers $u^{n}$ of the first cobordism Chern class. So their cartesian products $B_{i_{1}} \times \cdots \times B_{i_{k}}$ represent the basis elements $b_{i_{1}} \cdots b_{i_{k}}$ for $\Omega_{*}^{U}\left(B T^{k}\right)$, dual to monomials $u_{1}^{i_{1}} \cdots u_{k}^{i_{k}}$.

4) Hirzebruch genera. Classic genera associated to complex analytic manifolds include the Euler characteristic and Todd genus. These were extended to almost complex manifolds, and thence to complex cobordism classes, as they became expressible in terms of Chern numbers [59]. In modern parlance, a Hirzebruch genus is a homomorphism $f: \Omega_{*}^{U} \rightarrow R_{*}$ of graded rings into any commutative $R_{*}$, and is equivalent to a formal group law over $R_{*}[\mathbf{7 9}]$. Historically, $f$ was specified by a formal power series $t / e_{f}(t)$ in $R_{*} \otimes \mathbb{Q}[[t]]$, and $\left.e_{f}(t)\right)$ was identified with the exponential of the formal group law as soon as the concept was available [19], [80]. The construction and computation of genera and their equivariant analogues has a long and illustrious history $[\mathbf{4}],[\mathbf{5}],[\mathbf{3 9}],[\mathbf{5 8}],[\mathbf{6 0}],[\mathbf{6 5}],[\mathbf{6 6}],[\mathbf{6 8}],[\mathbf{8 1}]$.

A prime example is the universal toric genus $\Phi$, which may be evaluated on any $2 n$-dimensional $T^{k}$-manifold $(M, a)$ that carries a $T^{k}$-equivariant structure $c_{\nu}$ on its stable normal bundle. Then $\Phi\left(M, a, c_{\nu}\right)$ is given by the geometric cobordism class $\left[E T^{k} \times_{T^{k}} M \rightarrow B T^{k}\right]$ in $\Omega^{-2 n}\left(B T^{k}\right)$, and therefore takes the form

$$
\sum g_{i_{1} \cdots i_{k}} u_{1}^{i_{1}} \cdots u_{k}^{i_{k}}, \quad \text { where } g_{i_{1} \cdots i_{k}} \in \Omega_{2\left(n+i_{1}+\cdots+i_{k}\right)}^{U} .
$$

So $g_{0 \ldots 0}$ is precisely the cobordism class $[M]$ in $\Omega_{2 n}^{U}$, and we may represent $g_{i_{1} \ldots i_{k}}$ by a connected manifold that fibres over $B_{i_{1}} \times \cdots \times B_{i_{k}}$ with fibre $M$. Crucially, the base bounds, so we may relate the multiplicative properties of any Hirzebruch genus to the rigidity properties of its $T^{k}$-equivariant extension.

We may restrict attention to examples $\left(M, a, c_{\nu}\right)$ for which $a$ has isolated fixed points only, and $c_{\nu}$ is the complement of an equivariant structure $c_{\tau}$ on its stable tangent bundle; these include all toric and quasitoric manifolds. In order to interpret (4.5) in terms of local invariants at each fixed point $x$, we require the sign $\sigma(x)$ (in the alternative form above) and the weight vectors $w_{1}(x), \ldots, w_{n}(x)$ of the representation of $T^{k}$ in $T_{x}(M)$. The resulting localisation formula is

$$
\Phi\left(M, \alpha, c_{\tau}\right)=\sum_{x} \sigma(x) \prod_{j=1}^{n}\left(\left[w_{j}(x)\right]\left(u_{1}, \ldots, u_{n}\right)\right)^{-1}
$$

where $x$ ranges over the fixed point set of $a$ and $\left[w_{j}(x)\right]\left(u_{1}, \ldots, u_{j}\right)$ is the appropriate multivariable $w_{j}$-series for the universal formal group law. The fact that the irregular part of (4.6) must vanish imposes severe restrictions the possible signs and weight vectors. The formula reduces to the $S^{1}$ version of [25], and was obtained 
by Krichever [67] for almost complex manifolds (so $\sigma(x)=1$ ). It was applied in [26] to determine the complex bordism classes of classical homogeneous spaces. For quasitoric manifolds we have $k=n$, and the signs and weights may be described explicitly in terms of the combinatorial data $(P, \Lambda)$; the descriptions simplify significantly for projective toric varieties.

Masuda [70] and Panov [83] also obtained formulae evaluating Hirzebruch's original $\chi_{y}$-genus in terms of combinatorial data. Both confirmed the well-known fact that every smooth toric variety has Todd genus 1 , in contrast to the arbitrary integral values attainable on quasitoric manifolds.

5) CAT $(K)$-diagrams. The appropriate framework for the study of toric homotopy types is that of diagrams over the face category $\operatorname{CAT}(K)$, meaning covariant functors such as the exponential diagrams $X^{K} \rightarrow$ TOP of [86]. Here TOP denotes a category of pointed topological spaces such as $k$-spaces $[\mathbf{9 6}]$, of which $X$ is an object. Also, $X^{K}(\sigma)$ is the cartesian product $X^{\sigma}$, and $X^{K}(\sigma \subset \tau)$ is the inclusion $X^{\sigma} \rightarrow X^{\tau}$ induced by assigning the basepoint to the additional coordinates. In case $X$ is the classifying space $B T$ of the circle, colim ${ }_{\mathrm{TOP}} B T^{K}$ is precisely Buchstaber and Panov's construction $\bigcup_{\sigma \in K} B T^{\sigma}$ for $D J(K)[\mathbf{2 0}]$.

The corresponding descriptions of $\mathcal{Z}_{K}$ and its quotients is subtler - and more revealing. Consider the diagram $T^{V \backslash K}: \operatorname{CAT}(K) \rightarrow$ TOP, for which $T^{V \backslash K}(\sigma)$ is the torus $T^{V \backslash \sigma}$ and $T^{V \backslash K}(\sigma \subset \tau)$ is the projection $T^{V \backslash \sigma} \rightarrow T^{V \backslash \tau}$; then $\operatorname{colim}_{\mathrm{TOP}} T^{V \backslash K}$ is a point. There is a more complex diagram $D^{K}$, for which $D^{K}(\sigma)$ is the subspace $\left(D^{2}\right)^{\sigma} \times T^{V \backslash \sigma}$ of the product of 2-disks $\left(D^{2}\right)^{V}$, and $D^{V}(\sigma \subset \tau)$ is the corresponding inclusion $\left(D^{2}\right)^{\sigma} \times T^{V \backslash \sigma} \rightarrow\left(D^{2}\right)^{\tau} \times T^{V \backslash \tau}$. The projection $D^{K}(\sigma) \rightarrow T^{V \backslash K}(\sigma)$ is a homotopy equivalence for every $\sigma$, and induces a map colim ${ }_{\mathrm{TOP}} D^{K} \rightarrow \operatorname{colim}_{\mathrm{TOP}} T^{V \backslash K}$; yet colim ${ }_{\text {тор }} D^{K}$ is Buchstaber and Panov's construction for $\mathcal{Z}_{K}[\mathbf{2 0}]$. This example shows how badly colimits behave under objectwise homotopy equivalences, but also suggests a remedy. The homotopy colimit of $T^{V \backslash K}$ is defined by constructing a cofibrant replacement diagram such as $D^{K}$, and taking its ordinary colimit; in other words, the simpler diagram provides a weak equivalence hocolim ${ }_{\mathrm{TOP}} T^{V \backslash K} \simeq \mathcal{Z}_{K}$.

This procedure always works in favourable circumstances, although finding an appropriate cofibrant replacement may be difficult. For spaces $X$ such as $\mathrm{CW}$ complexes, the exponential diagram $X^{K}$ is already cofibrant, so we may also write $D J(K)$ as hocolim ${ }_{\text {Tор }} B T^{K}$. On the other hand, any quotient of $\mathcal{Z}_{K}$ by a subtorus $T^{l}<T^{V}$ gives rise to a weak equivalence hocolim ${ }_{\mathrm{TOP}} T^{V / l} \simeq \mathcal{Z}_{K} / T^{l}$, where $T^{V / l}(\sigma)$ is $T^{V \backslash \sigma} / \operatorname{Im}\left(T^{l}\right)$. In particular, this expresses any quasitoric manifold $M$ as a homotopy colimit, and extends the corresponding result [97, Proposition 5.3] for toric varieties, where homotopy colimits and toric geometry were first associated. The close relationship between derived forms and homotopy colimits actually hinges on the fact that the nerve of $\operatorname{CAT}(K)$ is the cone on the barycentric subdivision of $K$, and may therefore be identified with the polyhedral complex $P_{K}$.

Homotopy colimits over $\operatorname{CAT}(K)$ are sometimes preserved by the standard functors of algebraic topology, so long as they can be defined in the target category. The consequences of these ideas are studied in [85], along with many algebraic and geometrical examples associated to the vertex $T$.

6) Homotopy types. Explicit calculations of homotopy types fall into the three standard categories of increasing complexity; rational, $p$-adic, and integral. 
The rational homotopy type of any simply connected space $X$ is most efficiently encoded by Sullivan's minimal model, which is a well-structured commutative differential graded algebra that is quasi-isomorphic to the commutative cochain algebra $A_{P L}^{*}(X)[47]$. Spaces whose minimal models may be constructed directly from their rational cohomology algebras are known as formal, and play a central rôle in rational homotopy theory. Important examples include spheres, compact connected Lie groups, and compact Kähler manifolds.

The rationalisation of any $D J(K)$ or quasitoric manifold $M$ retains significant homotopy theoretic information. A result of $[\mathbf{7 6}]$ confirms that $D J(K)$ is formal for arbitrary $K$, and is applied in [85] to deduce the formality of $M$; indeed, the argument extends to cases such as the torus manifolds of [71]. By way of contrast, moment-angle complexes cannot generally be formal, as follows from Baskakov's discovery $[\mathbf{1 0}]$ of nontrivial Massey products in their rational cohomology for certain $K$. His calculations employed the bigraded chain complex of $\mathcal{Z}_{K}$ discussed in [20], and have since been extended by Denham and Suciu [38]. Minimal models for $D J(K)$ have been exhibited only for iterated joins of simplices and boundaries of simplices [76], in which case the cohomology ring is a complete intersection and uniquely determines the simply connected rational homotopy type.

So far as $p$-adic results are concerned, the $p$-completion of $D J(K)$ is uniquely determined by its mod- $p$ cohomology $\operatorname{ring} \mathbb{F}_{p}[K]$ whenever $K$ is a join of skeleta of simplices [77]. This holds for any prime $p$, and combines with the rational results to show integral uniqueness for complete intersections.

The integral homotopy type of the $\mathcal{Z}_{K}$ has long been of interest to combinatorialists, as we shall explain in our discussion of the face $A C T$. Work of Grbic and Theriault [52] showed that $\mathcal{Z}_{K}$ is weakly equivalent to a wedge of spheres whenever $K$ is obtained by iterated pushouts of shifted complexes. We may then interpret crucial elements of $\pi_{*}(D J(K))$ in terms of the homotopy quotient fibration

$$
T^{m} \longrightarrow \mathcal{Z}_{K} \longrightarrow D J(K) \text {. }
$$

In particular, $\pi_{*}(D J(K))$ contains many Whitehead products, both iterated and generalised, that directly reflect the combinatorial structure of $K$.

The possibility of analysing stable homotopy types has also been raised by [8], which considers homotopy decompositions of the suspensions $\Sigma \mathcal{Z}_{K}$ and their generalisations. These should be compared with earlier splittings of suspensions of $k$ th stage Bott towers into wedges of Thom complexes over lower stages [27], and analogous splittings for Dobrinskaya towers.

7) Oriented cohomology theories. The integral cohomology of any $D J(K)$ or quasitoric manifold $M^{2 n}$ is free, so evaluating a complex oriented cohomology theory $E^{*}(\cdot)$ on either space is relatively straightforward. Nevertheless, it is convenient to assume that the coefficient ring $\pi_{*} E=E_{*}$ is concentrated in even dimensions, and free of additive torsion. Then $E^{*}\left(D J(K)_{+}\right)$is isomorphic to the face ring $E_{*}[K]$, for example, because the corresponding Atiyah-Hirzebruch spectral sequence collapses. Given any simple polytope $P$ and dicharacteristic homomorphism $\ell: T^{\mathcal{F}} \rightarrow T^{n}$, the cohomology ring of the derived quasitoric manifold is given by an isomorphism

$$
E_{*}\left[K_{P}\right] / J_{E} \longrightarrow E^{*}\left(M(\ell)_{+}\right),
$$

where $J^{E}$ is the ideal generated by the first $E$-theory Chern classes of the $n$ complex line bundles of (4.2). These bundles are tensor products, so their Chern classes embody the formal group law associated to $E^{*}(\cdot)$. The resulting formulae are 
conceptually straightforward, and have been obtained independently by Strickland and the authors. Complex cobordism and complex $K$-theory are good examples, involving the universal and multiplicative formal group laws respectively, although detailed calculations with the former may require major technical expertise. One remarkable consequence is that the $\Omega_{*}^{U}$-module $\Omega_{*}^{U}(M)$ is generated by embedded quasitoric submanifolds for any quasitoric manifold $M$.

Computing the $K O$-theory of these spaces is much more difficult. A significant start was made by Bahri and Bendersky [7], who found a degree of order amidst the algebraic chaos of their Adams spectral sequence approach. In particular, they proved that $K O^{*}(M)$ is additively isomorphic to a sum of $K O_{*}$-modules of two types; namely $K O^{*}\left(S^{2 i}\right)$ for $0 \leq i \leq n$, and $K O^{*}\left(\Sigma^{2 j} \mathbb{C} P^{2}\right)$ for $0 \leq j \leq n-2$. The splitting depends only on the cohomology ring $H^{*}(M ; \mathbb{Z} / 2)$, and leads to the $B B$ numbers $p$ and $q$, which enumerate the summands of the first and second type respectively. More specific calculations were carried out in $[\mathbf{2 7}]$ for certain families of Bott towers, whose $K O_{*}$-algebra structures and $\mathrm{BB}$ numbers were determined.

The most general setting for these attempts is that of quaternionic oriented cohomology theory, for which quaternionic cobordism $\Omega_{S p}^{*}(\cdot)$ is the universal example. Neither the quaternionic cobordism ring nor the module structure of $\Omega_{S p}^{*}\left(\mathbb{C} P^{n}\right)$ are known, so any applications to toric spaces are extremely interesting, but well out of current reach.

\section{New Faces - and Beyond}

Having introduced the fourth vertex $T$, we conclude our invitation by advertising the seven new faces it defines, and speculating on future trends that should further increase the allure of the Tetrahedron.

The edge $\boldsymbol{A T}$. Even before the vertex $T$ was properly identified, several toric geometers had strayed along the edge $A T$ in their desire to investigate topological invariants of toric varieties. For example, Ehler's formula [43] for the Poincaré duals of the Chern classes of a nonsingular toric variety was utilised in Khovanskii's work [65] on the edge $A C$. His formula represents the dual of $c_{k}\left(\tau\left(M^{2 n}\right)\right)$ by the sum of the fundamental classes $\sum_{G} X(G)$ in $H_{2 n-2 k}\left(M_{+} ; \mathbb{Z}\right)$, taken over all the $2(n-k)$-dimensional facial submanifolds of $M$. It extends immediately to quasitoric manifolds, so long as $M$ is omnioriented and the $X(G)$ are invested with the induced omniorientations. Indeed, the formula may then be interpreted in $\Omega_{2 n-2 k}^{U}\left(M_{+}\right)$, and computes the the duals of the universal complex cobordism Chern classes.

Toric topologists have also visited $A T$, as exemplified by $[\mathbf{2 2}]$. The authors prove that any complex cobordism class may be represented by the quotient of a free torus action on a real quadratic complete intersection. The importance of the real quadratic viewpoint has recently been emphasised by Bosio and Meersseman [16], in their construction of families of non-Kähler complex manifolds using $\mathcal{Z}_{K}$.

The edge $\boldsymbol{C T}$. Exploration of the edge $C T$ motivated much of Buchstaber and Panov's survey [20]. Their results include a notable generalisation of the DehnSommerville equations (3.2), for which they applied Poincaré duality to prove

$$
h_{j}-h_{n-j}=(-1)^{n-j}\left(\chi(K)-\chi\left(S^{n-1}\right)\right)\left(\begin{array}{l}
n \\
j
\end{array}\right)
$$

for any triangulated $(n-1)$-manifold $K$, and $1 \leq j \leq n$. It follows that the $h_{j}-h_{n-j}$ are homotopy invariants, and independent of the triangulation. 
This edge is also a natural habitat for Halperin's well-known toral rank conjecture, concerning the largest integer $\operatorname{trk}(X)$ for which a finite-dimensional topological space $X$ admits an almost free $T^{\operatorname{trk}(X)}$-action. Every isotropy subgroup is necessarily finite, and the conjecture states that $\operatorname{dim} H^{*}\left(X_{+} ; \mathbb{Q}\right) \geq 2^{\operatorname{trk}(X)}$. If the conjecture holds, then it follows from $[\mathbf{2 1}]$ that any $(n-1)$-dimensional simplicial complex $K$ on vertices $[m]$ satisfies the inequality

$$
\operatorname{dim}\left(\bigoplus H^{*}\left(K_{\omega} ; \mathbb{Q}\right)\right) \geq 2^{m-n}-1
$$

where $\omega$ ranges over the subsets of $[m]$, and the $K_{\omega}$ are induced subcomplexes. When $K$ is the boundary of $\Delta^{n}$, for example, the right hand side of (5.1) is 1 , and equality holds. Computer calculations confirming the exponential growth required by the conjecture for cyclic polytopes are currently in progress by Gadjikurbanov.

The edge $\boldsymbol{S T}$. The edge $S T$ is becoming densely populated, as realisation dawns that global properties of Hamiltonian actions are as much a part of equivariant topology as of the symplectic geometry that nurtured their growth. This viewpoint is expounded by Guillemin, Ginzburg and Karshon [55], who provide a beautiful survey of many exciting aspects of $S T$, and initiate several new ideas that stray into adjacent faces of the Triangle.

For example, the famous Duistermaat-Heckman formula [42]

$$
\int_{M} e^{\langle\Phi, \xi\rangle} \omega^{n}=(2 \pi)^{n} n ! \sum_{x \in M^{T}} e^{\langle\Phi(x), \xi\rangle} \prod_{j=1}^{n}\left\langle w_{j}(x), \xi\right\rangle^{-1}
$$

is associated to the process of symplectic reduction, and is well known to mathematical physicists as the exact stationary phase formula. Atiyah and Bott and Berligne and Vergne proved that it is also a special case of a localisation formula of equivariant cohomology that arises in the presence of isolated fixed points; further explanation is given in [55], for example. From this viewpoint, (5.2) is closely related to the universal toric genus (4.6), as is evident from their formal similarity.

These considerations are relevant to the topology of symplectic circle-actions on a compact symplectic $M$. In dimension 4 , McDuff proved that any such action is Hamiltonian if and only if the fixed point set is nonempty, and gave a 6-dimensional non-Hamiltonian example whose fixed point sets are 2-tori. Subsequently, Feldman [46] studied the case of isolated fixed points, and deduced that the Todd genus of $M$ is necessarily 1 or 0 ; if 1 , the action is Hamiltonian, and if 0 , it is not.

The facet $\boldsymbol{A C T}$. This facet is home to the development of a special class of subspace arrangements, as introduced on $A C S$; here too, work represents the limit of activity within the interior of $T T$.

For any subset $\sigma \subseteq[m]$ we write the coordinate subspace $\left\{z: z_{j}=0\right.$ if $\left.j \in \sigma\right\}$ as $L_{\sigma} \leq C^{m}$ (otherwise known as $\mathbb{C}^{[m] \backslash \sigma}$ in Example 2.1). So an arbitrary simplicial complex $K$ on vertices $[m]$ determines the coordinate subspace arrangement $\left\{L_{\sigma}: \sigma \notin K\right\}$, with complement $U(K)$. So $T^{m}$ acts on $U(K)$ coordinatewise, and we may interpret $U$ globally as a diagram of $T^{m}$-spaces on the category of subcomplexes of the $(m-1)$ simplex on vertices $[m]$, for every $m>0$. The relevance of $U$ to $T T$ is epitomised by [20, Theorem 8.9], which establishes a natural $T^{m}$-equivariant deformation retraction $U(K) \rightarrow \mathcal{Z}_{K}$. In other words, we may study homotopy theoretic properties of moment-angle complexes purely in terms of coordinate subspace arrangements. In particular, we obtain smooth structures on the $\mathcal{Z}_{K}$, 
Well before this link was established, Ziegler and Živaljević [101] brought the theory of homotopy colimits to bear, and rederived Goresky and MacPherson's algebraic decomposition of $H^{*}(U(A) ; \mathbb{Z})$. For the case $U(K)$, their ideas lie behind our current formulation of $\mathcal{Z}_{K}$ as a homotopy colimit over CAT $(K)$. For coordinate subspace arrangements, the decomposition of $H^{*}(U(K) ; \mathbb{Z})$ was also recovered in [11] by applying a theorem of Hochster, and the link with $\mathcal{Z}_{K}$ was exploited to deduce the multiplicative structure; similar results were obtained independently by Franz [48]. The algebraic decomposition has recently been realised topologically, by splitting $\Sigma \mathcal{Z}_{K}[\mathbf{8}]$.

Further techniques of unstable homotopy theory are now being focused on moment-angle complexes in this context. For example, iterated and generalised Samelson products in the loopspaces $\Omega \mathcal{Z}_{K}$ are under consideration, and their relationship with the geometry of the arrangement and the combinatorics of $K$ promises to be particularly fruitful.

Other questions that were originally posed in the era of torus embeddings have also been formulated and solved in the context of the facet $A C T$. One such is the weak Oda conjecture of 1978, concerning the factorisation of proper equivariant birational maps of nonsingular toric varieties into sequences of well-behaved blow-ups and blow-downs. The conjecture may be approached using combinatorial topology, because any bistellar move on the simplicial complex $K_{\Sigma}$ corresponds to a combinatorial operation on the fan $\Sigma$, and thence to a blow-up and blow-down on $X_{\Sigma}$. It was verified in 1997 by Morelli and Włodarczyk [98], who proved that any two regular fans in $\mathbb{R}^{n}$ are connected by a sequence of bistellar operations through regular fans. The result was then extended to other nonsingular varieties by a process of torification [1].

The facet $\boldsymbol{A S T}$. The facet $A S T$ may not yet have acquired a distinctive literature, but two topics suggest themselves as candidates.

For any symplectic manifold $(M, \omega)$, let $\operatorname{Diff}(M)$ denote the group of diffeomorphisms of the underlying smooth manifold, and consider the subgroups

$$
\operatorname{Ham}(M, \omega)<\operatorname{Sym}(M, \omega)<\operatorname{Diff}(M)
$$

of Hamiltonian diffeomorphisms and symplectomorphisms respectively. Algebraic, geometric and homotopy theoretic properties of these groups are discussed in McDuff's survey of 2003 [72], but many questions remain open. It is therefore natural to consider examples in which $M$ admits the action of a compact Lie group [63]; in particular, the situation for toric varieties and quasitoric manifolds has aroused recent interest.

Harada and Landweber's study [57] of the equivariant $K$-theory of Hamiltonian $T^{n}$-spaces should also be located in this facet.

The facet $\boldsymbol{C S T}$. A beautiful circle of ideas that typify the facet $C S T$ arises from the action by conjugation of the unitary group $U(n)$ on the vector space space $H$ of $n \times n$ Hermitian matrices. The orbit $H_{\lambda}$ of the diagonal matrix $d\left(\lambda_{1}, \ldots, \lambda_{n}\right)$ is diffeomorphic to the $\left(n^{2}-n\right)$-dimensional flag manifold of complete flags in $\mathbb{C}^{n}$, and admits a standard symplectic structure. The natural left action of $T^{n}<U(n)$ is Hamiltonian, and we may identify the corresponding moment map with the restriction to $H_{\lambda}$ of $\Phi: H \rightarrow \mathbb{R}^{n}$, which associates to any Hermitian matrix its vector of diagonal entries. A classical theorem of Schur and Horn $[\mathbf{6}]$ asserts that the image $\Phi\left(H_{\lambda}\right)$ is the convex hull of the $n$ ! points obtained by permuting the 
coordinates of $\left(\lambda_{1}, \ldots, \lambda_{n}\right)$ in $\mathbb{R}^{n}$. The resulting simple convex polytope $\Pi^{n-1}$ is the famous permutahedron [99], and a section for $\Phi$ is given by lifting each of its points $p$ to the corresponding diagonal matrix $d(p)$.

Now consider the manifold $J_{\lambda}^{2(n-1)}$ of $n \times n$ tridiagonal Hermitian matrices, with distinct eigenvalues $\lambda_{1}, \ldots, \lambda_{n}$. According to [15], there is a non-standard embedding $J_{\lambda}^{2(n-1)} \subset H_{\lambda}$ that serves as a symplectic reduction; the restriction of the symplectic form on $H_{\lambda}$ is non-degenerate, and the restriction of the moment map is a completely integrable system. In particular, $J_{\lambda}^{2(n-1)}$ is a toric and quasitoric manifold over $\Pi^{n-1}$. Furthermore, the permutahedron is the polar of the barycentric subdivision of $\Delta^{n-1}$, and is therefore $(n-1)$-colourable. It follows from [35, Corollary 6.10] that $J_{\lambda}^{2(n-1)}$ is stably parallelisable, and hence of serious interest to stable homotopy theorists.

The interior. The interior of $T T$ is relatively uncharted, but exploratory settlements have been established and several further expeditions are under way.

Panov's work [84] is a recent example, which aims to exploit the relative ease with which topologists construct the factorisation $\mathcal{Z}_{K} \rightarrow M \rightarrow P_{K}$ of (2.2) for any quasitoric manifold $M$. Analogues for algebraic varieties and completely integrable torus actions were obtained by several authors in the early 1990s, as described by Cox $[\mathbf{3 0}]$. The results may be interpreted as providing homogeneous coordinates for the varieties in question, but depend on the notions of categorical quotient and symplectic reduction respectively. Panov achieved a more satisfying unification by adapting the concept of Kempf-Ness set from the theory of algebraic group actions on affine varieties, and applying it to the coordinate subspace pair $\left(U(K), \mathcal{Z}_{K}\right)$. His procedure works for toric orbifolds, and is closest to the motivating example in the case of projective toric varieties.

A more established exploration of the interior concerns the pursuit of mirror symmetry, first observed by theoretical physicists. Originally formulated as the identification of partition functions on a pair of Calabi-Yau manifolds $\left(V, V^{*}\right)$, the subject became intertwined with toric geometry under the influence of Batyrev, as summarised in his seminal paper [12], for example. In 1999, Cox and Katz published an entertaining survey [32] from the algebraic viewpoint.

A convex polytope $P$ in $\mathbb{R}^{n}$ is reflexive if it is integral, contains the origin in its interior, and has integral polar $P^{*}$; then $P^{*}$ is also reflexive. Following the construction of [64] in the case of a single generic hypersurface, Batyrev conjectured that the corresponding pair $\left(X_{P}, X_{P^{*}}\right)$ of toric varieties is mirror symmetric. By defining stringy Hodge numbers for reasonable singular varieties, he proposed an extension of the simplest topological test for smooth pairs $\left(V, V^{*}\right)$ to be mirror symmetric, and proved that any $\left(X_{P}, X_{P^{*}}\right)$ satisfies his test. Work on his conjectures is ongoing. An inspection of the bibliography of $[49]$ confirms that the subject lies close to the the facet $A C S$; nevertheless, it has a small but significant $T$-coordinate.

Many examples in mirror symmetry involve toric orbifolds, which arise algebraically from a class of simplicial fans and topologically from characteristic functions that fail to satisfy Davis and Januszkiewicz's condition $(*)$.

ExAmple 5.1. Following Example 2.2, let $Z$ be $S^{2 n+1} \subset \mathbb{C}^{n+1}$, and $a$ the coordinatewise action of $T^{n+1}$. For any vector $\chi$ of positive integers $\left(\chi_{1}, \ldots, \chi_{n+1}\right)$, let $H$ be the subcircle $T(\chi)<T^{n+1}$ of points

$$
\left\{\left(t^{\chi_{1}}, \ldots, t^{\chi_{n+1}}\right): t \in T\right\}
$$


and $M$ the quotient space $S^{2 n+1} / T(\chi)$; so $M$ is acted on by the quotient $n$-torus $T_{\chi}=T^{n+1} / T(\chi)$. In this case $M$ is the weighted projective space $\mathbb{C} P^{n}(\chi)$, and reduces to the standard projective space $\mathbb{C} P^{n}$ in case $\chi=(1, \ldots, 1)$.

Weighted projective spaces have inspired an impressive literature of their own, with links to each of the vertex disciplines; [41] is a well-known survey. Recent work is increasingly topological, and includes the calculation [9] of the equivariant cohomology ring $H_{T_{\chi}}^{*}\left(\mathbb{C} P^{n}(\chi) ; \mathbb{Z}\right)$; this doubles as a face ring for the weighted quotient simplex of [69], and is described by generators and relations in terms of piecewise polynomials on the associated fan. It is also isomorphic to the Chow ring of $\mathbb{C} P^{n}(\chi)$.

Further research into the algebraic topology of quasitoric orbifolds is under active development, and includes applications of weighted lens spaces, homotopy colimits, weighted face rings, and the Bousfield Kan spectral sequence, together with calculations in $K$-theory and cobordism. The topic certainly appears to merit inclusion deep within the interior of the Tetrahedron.

So far as future trends are concerned, applications to the motion planning problem of robotics look especially intriguing. Moment-angle complexes $\mathcal{Z}_{K}$ are known to engineers as configuration spaces of planar linkages [61], and their topological complexity measures the instability of algorithms for determining paths between initial and final states of the system [45]. Bounds on the topological complexity depend on the homotopy type and cohomological structure of $\mathcal{Z}_{K}$, and are therefore within reach for certain specific $K$.

Beyond TT. Experience shows that toric objects provide concrete realisations of abstract concepts in all four vertex disciplines, which not only stimulate the interests of experts, but are also attractive and comprehensible to a general mathematical audience. Such reasons suggest that the study of $T T$ will continue to flourish. We therefore extend one final invitation to future authors, to engage in the light-hearted but enlightening exercise of determining the toric coordinates of their own lectures and publications.

It seems likely that $T T$ will eventually be subdivided as areas acquire sufficient theory, literature, and distinction to merit a separate identity. But we cannot imagine notation to accommodate this process! It is also fascinating to speculate on whether additional vertices lie buried in the future - although mathematical and theoretical physicists' increasing involvement suggests that vertex $P$ may soon emerge. We encourage readers to dream of a Toric Four-Simplex, and entertain us with their fantasies at future toric conferences ...

\section{References}

[1] Dan Abramovich, Kalle Karu, Kenji Matsuki, and Jarosław Włodarczyk. Torification and factorization of birational maps. Journal of the American Mathematical Society 15 (2002) 531-572.

[2] Vladimir I Arnold. The cohomology ring of the colored braid group. Mathematical Notes 5 (1969) 138-140.

[3] Michael F Atiyah. Convexity and commuting Hamiltonians. Bulletin of the London Mathematical Society 14 (1982) 1-15.

[4] Michael F Atiyah and Friedrich Hirzebruch. Spin-manifolds and group actions. Essays on Topology and Related Topics (Mémoires dédiés à Georges de Rham), Springer (1970) 18-28.

[5] Michael F Atiyah and Isadore M Singer. The index of elliptic operators I. Annals of Mathematics 87(2) (1968) 484-530. 
[6] Michéle Audin. Torus Actions on Symplectic Manifolds. 2nd revised edition. Progress in Mathematics 93, Birkhäuser (2004).

[7] Anthony Bahri and Martin Bendersky. The KO-theory of toric manifolds. Transactions of the American Mathematical Society 352 (2000) 1191-1202.

[8] Anthony Bahri, Martin Bendersky, Frederick R Cohen, and Samuel Gitler. The polyhedral product functor: a method of computation for moment-angle complexes, arrangements and related spaces. arXiv:math 0711.4689v1 (2007).

[9] Anthony Bahri, Matthias Franz, and Nigel Ray. The equivariant cohomology of weighted projective spaces. arXiv:math 0708.1581v2 (2007).

[10] Ilia V Baskakov. Massey triple products in the cohomology of moment-angle complexes. Russian Mathematical Surveys 58(5) (2003) 1039-1041.

[11] Ilia V Baskakov, Victor M Buchstaber, and Taras E Panov Cellular cochain algebras and torus actions. Russian Mathematical Surveys 59(3) (2004) 562-563.

[12] Victor V Batyrev. Mirror symmetry and toric geometry. Documenta Mathematica (Extra Volume ICM 1998 II) 239-248.

[13] Louis J Billera and Carl W Lee. Sufficiency of McMullen's conditions for f-vectors of simplicial polytopes. Bulletin of the American Mathematical Society 2 (1980) 181-185.

[14] Anders Björner. Subspace arrangements. First ECM Volume I (Paris, 1992), Progress in Mathematics 119, Birkhuser (1994) 321-370.

[15] Anthony M Bloch, Hermann Flaschka, and Tudor Ratiu. A convexity theorem for isospectral manifolds of Jacobi matrices in a compact Lie algebra. Duke Mathematical Journal 61(1) (1990) 1, 41-65.

[16] Frédéric Bosio and Laurent Meersseman. Real quadrics in $\mathbb{C}^{n}$, complex manifolds and convex polytopes. Acta Mathematica 197(1) (2006) 53-127.

[17] Arne Brøndsted. An Introduction to Convex Polytopes. Graduate Texts in Mathematics 90, Springer (1983).

[18] Winfried Bruns and Hans Jørgen Herzog. Cohen-Macaulay Rings. Cambridge Studies in Advanced Mathematics 39, CUP (1998).

[19] Victor M Buchstaber. The Chern-Dold character in cobordism I. Math.USSR Sbornik, 12:4, 1970, 573-594.

[20] Victor M Buchstaber and Taras E Panov. Torus Actions and their Applications in Topology and Combinatorics. University Lecture Series 24, AMS (2002).

[21] Victor M Buchstaber and Taras E Panov. Combinatorics of simplicial cell complexes, and torus actions Proceedings of the Steklov Mathematical Institute 247 (2004) 1-17.

[22] Victor M Buchstaber, Taras Panov, and Nigel Ray. Spaces of polytopes and cobordism of quasitoric manifolds. Moscow Mathematical Journal 7(2) (2007) 219-242.

[23] Victor M Buchstaber and Nigel Ray. Toric manifolds and complex cobordisms. Russian Mathematical Surveys 53(2) (1998) 371-373.

[24] Victor M Buchstaber and Nigel Ray. Tangential structures, toric manifolds, and connected sums of polytopes. International Mathematics Research Notices 4 (2001) 193-219.

[25] Victor M Buchstaber and Nigel Ray. The universal equivariant genus and Krichever's formula. Russian Mathematical Surveys 62(1) (2007) 178-180.

[26] Victor M Buchstaber and Svjetlana Terzić. Equivariant complex structures on homogeneous spaces and their cobordism classes. arXiv:math 0708.2054v2 (2007).

[27] Yusuf Civan and Nigel Ray. Homotopy decompositions and real K-Theory of Bott towers. $K$-Theory 34 (2005) 1-33.

[28] Pierre E Conner and Edwin E Floyd. Torsion in SU-Bordism. Memoirs of American Mathematical Society 60, AMS (1966).

[29] David A Cox. The homogeneous coordinate ring of a toric variety. Journal of Algebraic Geometry 4 (1995) 17-50.

[30] David A Cox. Recent developments in toric geometry. In Algebraic Geometry - Santa Cruz 199. Proceedings of Symposia in Pure Mathematics 62(2), AMS (1997) 389-436.

[31] David A Cox. Update on toric geometry. Seminares and Congress 6 (2002) 1-41.

[32] David A Cox and Sheldon Katz. Mirror Symmetry and Toric Geometry. Mathematical Surveys and Monographs 68, AMS (1999).

[33] Vladimir I Danilov. The geometry of toric varieties. Russian Mathematical Surveys 33(2) (1978) 97-154. 
[34] Michael Davis. Smooth G-manifolds as collections of fiber bundles. Pacific Journal of Mathematics 77(2) (1978) 315-363.

[35] Michael W Davis and Tadeusz Januszkiewicz. Convex polytopes, Coxeter orbifolds and torus actions. Duke Mathematical Journal 62(2) (1991) 417-451.

[36] Thomas Delzant. Hamiltoniens périodiques et image convexe de l'application moment. Bulletin de la Société mathématique de France 116 (1988) 315-339.

[37] Michel Demazure. Sous-groupes algébriques de rang maximum du groupe de Cremona. Annales Scientifiques de lÉcole Normale Supérieure. 3(4) (1970) 507-588.

[38] Graham Denham and Alexander I Suciu. Moment-angle complexes, monomial ideals, and Massey products. Pure and Applied Mathematics Quarterly 3(1) (2007) 25-60.

[39] Tammo tom Dieck. Bordism of $G$-manifolds and integrality theorems. Topology 9 (1970) $345-358$.

[40] Natalia Dobrinskaya. The classification problem for quasitoric manifolds over a given polytope. Functional Analysis and its Applications 35(2) (2001) 83-89.

[41] Igor Dolgachev. Weighted projective varieties, Group actions and vector fields (Vancouver BC, 1981). Lecture Notes in Mathematics 956, Springer (1982) 34-71.

[42] Hans Duistermaat and Gert Heckman. On the variation in the cohomology of the symplectic form of the reduced phase space. Inventiones Mathematicae 69 (1982) 153-158 and Addendum 72 (1983) 259-269.

[43] Fritz Ehlers. Eine Klasse komplexer Mannigfaltigkeiten und die Auflösung einiger isolierter Singularitäten. Mathematische Annalen 218 (1975) 127-156.

[44] Günter Ewald. Combinatorial Convexity and Algebraic Geometry. Graduate Texts in Mathematics 168, Springer (1996).

[45] Michael Farber. Topological complexity of motion planning. Discrete Computational Geometry 29 (2003) 211-221.

[46] Konstantin E Feldman. Hirzebruch genera of manifolds equipped with a Hamiltonian circle action. Russian Mathematical Surveys 56(5) (2001) 978-979.

[47] Yves Felix, Stephen Halperin and Jean-Claude Thomas. Rational Homotopy Theory. Graduate Texts in Mathematics 205, Springer (2001).

[48] Matthias Franz. On the integral cohomology of toric varieties. Proceedings of the Steklov Institute of Mathematics 252 (2006) 53-62.

[49] Kenji Fukaya, Yong-Geun Oh, Kaoru Ono and Gang Tian (Editors) Symplectic Geometry and Mirror Symmetry: Proceedings of the 4 th KIAS Annual International Conference Seoul, August 2000. World Scientific Publishing Company (2001).

[50] William Fulton. Introduction to Toric Varieties. Annals of Mathematics Studies 131, Princeton University Press, Priceton NJ (1993).

[51] Mark Goresky and Robert MacPherson. Stratified Morse Theory. Springer (1988).

[52] Jelena Grbić and Stephen Theriault. The homotopy type of the complement of a coordinate subspace arrangement. Topology 46 (2007) 357-396.

[53] Michael Grossberg and Yael Karshon. Bott towers, complete integrability, and the extended character of representations. Duke Mathematical Journal 76 (1994) 23-58.

[54] Branko Grünbaum. Convex Polytopes. 2nd edition. Graduate Texts in Mathematics 221, Springer (2003).

[55] Victor Guillemin, Viktor Ginzburg, and Yael Karshon. Moment Maps, Cobordisms, and Hamiltonian Group Actions. Mathematical Surveys and Monographs 98, AMS (2002).

[56] Victor Guillemin and Shlomo Sternberg. Convexity properties of the moment mapping. Inventiones Mathematicae 67(3) (1982) 491-513.

[57] Megumi Harada and Gregory D Landweber. The K-theory of abelian symplectic quotients. arXiv:math 0612660v3 (2008)

[58] Akio Hattori. Spin-structures and $S^{1}$-actions. Inventiones Mathematicae 48 (1978) 7-36.

[59] Friedrich Hirzebruch. Topological Methods in Algebraic Geometry. 3rd edition. Springer (1966).

[60] Friedrich Hirzebruch, Thomas Berger, and Rainer Jung. Manifolds and Modular Forms. Max-Planck-Institut für Mathematil, Bonn, 1992.

[61] Ivan V Izmestiev. Manifolds determined by simple polyhedra as configuration spaces of hinge mechanisms. Russian Mathematical Surveys 55(1) (2000) 176-177.

[62] Klaus Jänich. On the classification of $O(n)$-manifolds. Mathematische Annalen 176 (1968) $53-76$. 
[63] Jatek Kẹdra and Dusa McDuff. Homotopy properties of Hamiltonian group actions. Geometric Topology 9 (2005) 121-162.

[64] Askold G Khovanskii. Newton polyhedra and toric varieties. Functional Analysis and its Applications 11 (1977) 56-67.

[65] Askold G Khovanskii. Newton polyhedra and the genus of complete intersections. Functional Analysis and its Applications 12 (1978) 38-46.

[66] Igor M Krichever. Obstractions to the existence of $S^{1}$-actions. Bordism of ramified covering. Izvestija Akademii Nauk SSSR 10(4) (1976) 783-797.

[67] Igor M Krichever. Formal groups and the Atiyah-Hirzebruch formula. Izvestiya Akademii Nauk SSSR 38 (1974) 1289-1304.

[68] Igor M Krichever. Generalised elliptic genera and Baker-Akhiezer functions. Mathematical Notes 47(1-2) (1990) 132-142.

[69] Eugene Lerman and Susan Tolman. Hamiltonian torus actions on symplectic orbifolds and toric varieties. Transactions of the American Mathematical Society 349 (1997) 4201-4230.

[70] Mikiya Masuda. Unitary toric manifolds, multi-fans and equivariant index. Tohoku Mathematical Journal 51(2) (1999) 237-265.

[71] Mikiya Masuda and Taras Panov. On the cohomology of torus manifolds. Osaka Journal of Mathematics 43 (2006) 711-746.

[72] Dusa McDuff. Lectures on groups of symplectomorphisms. arXiv:math 0201032v2 (2003).

[73] Peter McMullen. The numbers of faces of simplicial polytopes. Israel Journal of Mathematics 9 (1971) 559-570.

[74] John W Milnor. Construction of universal bundles I,II. Annals of Mathematics 63(2) (1956) 272-284, 430-436.

[75] John W Milnor. On the cobordism ring $\Omega^{*}$ and a complex analogue I. American Journal of Mathematics 82(3) (1960) 505-521.

[76] Dietrich Notbohm and Nigel Ray. On Davis-Januszkiewicz homotopy types I; formality and rationalisation. Algebraic \& Geometric Topology 5 (2005) 31-51.

[77] Dietrich Notbohm and Nigel Ray. On Davis-Januszkiewicz homotopy types II; completion and globalisation. Preprint (2008): available at www.ma.manchester.ac.uk

[78] Sergei P Novikov. Some problems in the topology of manifolds connected with the theory of Thom spaces. Doklady Akademii Nauk SSSR 132(5) (1960) 1031-1034.

[79] Sergei P Novikov. The methods of algebraic topology from the viewpoint of cobordism theory. Izvestiya Akademii Nauk SSSR 1(4) (1967) 827-913.

[80] Sergei P Novikov. Adams operators and fixed points. Izvestiya Akademii Nauk SSSR 2(6) (1968) 1193-1211.

[81] Serge Ochanine. Sur les genres multiplicatifs définis par des intgrales elliptiques. Topology 26(2) (1987) 143-151.

[82] Tadao Oda. Convex Bodies and Algebraic Geometry: An Introduction to the Theory of Toric Varieties. Springer, New York (1988).

[83] Taras Panov. Hirzebruch genera of manifolds with torus actions. Izvestiya: Mathematics 65(3) (2001) 543-556.

[84] Taras Panov. The topology of Kempf-Ness sets for algebraic torus actions. arXiv:math 0603556v2 (2006).

[85] Taras Panov and Nigel Ray. Categorical aspects of toric topology. In this volume; arXiv:math /0707.0300 (2007).

[86] Taras Panov, Nigel Ray, and Rainer Vogt. Colimits, Stanley-Reisner algebras, and loop spaces. In Algebraic Topology Categorical Decomposition Techniques, edited by Greg Arone, Ran Levi, and Michael Weiss. Progress in Mathematics 215, Birkhäuser (2004) 261-291.

[87] Daniel Quillen. Elementary proofs of some results of cobordism theory using Steenrod operations. Advances in Mathematics 7 (1971) 29-56.

[88] Nigel Ray. On a construction in bordism theory. Proceedings of the Edinburgh Mathematical Society 29 (1986) 413-422.

[89] Gerald A Reisner. Cohen-Macauley quotients of polynomial rings. Advances in Mathematics 21(1) (1976) 30-49.

[90] Marcel-Paul Schützenberger. A characteristic property of certain polynomials of EF Moore and CE Shannon. RLE Quarterly Progress Report 55, Reseearch Laboratory of Electronics, MIT (1959) 117-118. 
[91] Richard Stanley. The number of faces of a simplicial complex polytope. Advances in Mathematic 35 (1980) 236-238.

[92] Richard Stanley. Combinatorics and Commutative Algebra. 2nd edition. Progress in Mathematics 41, Birkhäuser (1996).

[93] Ernst Steinitz. Über die Eulerschen Polyederrelationen. Arxiv für Mathematik und Physik 11 (1906) 86-88.

[94] Bernd Sturmfels and Seth Sullivant. Toric geometry of cuts and splits. arXiv:math 0606683v2 (2007).

[95] Ernest B Vinberg. Discrete linear groups that are generated by reflections (Russian). Izvestija: Mathematics 35 (1971) 1072-1112.

[96] Rainer M Vogt. Convenient categories of topological spaces for homotopy theory. Archiv der Mathematik 22 (1971) 545-555.

[97] Volkmar Welker, Günter M Ziegler, and Rade T Živaljević. Homotopy colimits - comparison lemmas for combinatorial applications. Journal für die reine und angewandte Mathematik 509 (1999) 117-149.

[98] Jarosław Włodarczyk. Decomposition of birational toric maps in blow-ups \& blow-downs. Transactions of the American Mathematical Society 349(1) (1997) 373-411.

[99] Günter M Ziegler. Lectures on Convex Polytopes. Graduate Texts in Mathematics 152, Springer (1995).

[100] Günter M Ziegler. Face numbers of 4-polytopes and 3-spheres. Proceedings of the ICM 2002 Beijing, Volume III (2004) 625-634.

[101] Günter M Ziegler and Rade T Živaljević. Homotopy types of subspace arrangements via diagrams of spaces. Mathematische Annalen 295(3) (1993) 527-548.

Steklov Mathematical Institute, Russian Academy of Sciences, Gubkina Street, Moscow 119991, Russia

E-mail address: buchstab@mi.ras.ru

School of Mathematics, The University of Manchester, Oxford Road, Manchester M13 9PL, ENGLAND

E-mail address: victor.buchstaber@manchester.ac.uk

School of Mathematics, The University of Manchester, Oxford Road, Manchester M13 9PL, ENGLAND

E-mail address: nigel.ray@manchester.ac.uk 